\title{
Optimal Periodic Dividend and Capital Injection Problem for Spectrally Positive Lévy Processes
}

\author{
Yongxia Zhao ${ }^{\mathrm{a}, *}$, Ping Chen ${ }^{\mathrm{b}}$, Hailiang Yang ${ }^{\mathrm{c}}$ \\ ${ }^{a}$ School of Statistics, Qufu Normal University, Qufu, Shandong, China \\ ${ }^{b}$ Faculty of Business and Economics, University of Melbourne, Melbourne, Australia \\ ${ }^{c}$ Department of Statistics and Actuarial Science, The University of Hong Kong, Hong Kong
}

\begin{abstract}
In this paper, we investigate an optimal periodic dividend and capital injection problem for spectrally positive Lévy processes. We assume that the periodic dividend strategy has exponential inter-dividend-decision times and continuous monitoring of solvency. Both proportional and fixed transaction costs from capital injection are considered. The objective is to maximize the total value of the expected discounted dividends and the penalized discounted capital injections until the time of ruin. By the fluctuation theory of Lévy processes in Albrecher et al. (2016), the optimal periodic dividend and capital injection strategies are derived. We also find that the optimal return function can be expressed in terms of the scale functions of Lévy processes. Finally, numerical examples are studied to illustrate our results.

Keywords: Periodic dividend, Capital injection, Lévy process, Stochastic control, Scale function.

JEL: C44, C61, G32, G35.
\end{abstract}

\section{Introduction}

The Lévy risk model with positive jumps (spectrally positive Lévy process), which is also called dual risk model, is proposed to offset continuous expenses by the stochastic and irregular gains. Examples include research-based or commissionbased companies. In this context, dividend optimization problems have attracted extensive attention. In Avanzi et al. (2007) and Avanzi and Gerber (2008), the authors studied how the expectation of the discounted dividends until ruin can be calculated in the dual compound Poisson risk model. In Kyprianou et al. (2012),

\footnotetext{
${ }^{*}$ Corresponding author

Email addresses: yongxiazhao@163.com (Yongxia Zhao), pche@unimelb.edu.au (Ping Chen), hlyang@hku.hk (Hailiang Yang)
} 
Bayraktar et al. (2013), Yin and Wen (2013) and Zhao et al. (2015), the optimal dividend problems were studied in a general spectrally positive Lévy risk model.

In the above papers, the dividend decisions are made continuously, which usually leads to very irregular dividend payments. However, in practice, the companies that are capable of distributing dividends make dividend decisions on a periodic basis. Albrecher et al. (2011a) studied the random inter-dividend-decision times in the Cramér-Lundberg model, where the ruin can not occur between dividend payment times. Continuous monitoring of solvency with periodic dividends were considered by Albrecher et al. (2011b) in the Brownian risk model, Avanzi et al. (2013) and Avanzi et al. (2014) in the dual compound Poisson model. Recently, when the interdividend-decision times of periodic dividend are exponential, Pérez and Yamazaki (2016) showed the optimality of periodic barrier strategy for spectrally positive Lévy processes.

When dividend payments are maximized, ruin is usually certain. In some cases, it may be profitable to rescue the company by capital injection. This idea goes back to Porteus (1977). Yao et al. (2011) considered an optimal dividend and capital injection problem in the dual compound Poisson risk model. Avanzi et al. (2011) discussed the same problem in the dual compound Poisson model with diffusion. Bayraktar et al. (2013) and Zhao et al. (2015) extended their work to general spectrally positive Lévy processes. In addition, transaction cost, which usually includes two parts: proportional cost and fixed cost, is an important factor in business activities. In Avanzi et al. (2011), the proportional transaction costs from dividend and capital injection were involved into an optimal dividend problem. In Yao et al. (2011) and Zhao et al. (2015), both proportional and fixed costs on capital injection were considered. Fixed costs on dividend were studied in Bayraktar et al. (2014).

In this paper, the optimal periodic dividend and capital injection problem is discussed for spectrally positive Lévy processes. For periodic dividend, we assume that the inter-dividend-decision times are exponential as in Avanzi et al. (2014) and Pérez and Yamazaki (2016), but different methods are used. For capital injection, we include the proportional and fixed transaction costs. We also assume that the ruin may occur even under the rescue of capital injection. Like Zhao et al. (2015), we decompose the optimal problem into two suboptimal problems. By the fluctuation theory of Lévy processes observed at Poisson arrival in Albrecher et al. (2016), we find the optimal strategy and the optimal return function. If the fixed transaction cost from capital injection tends to zero, we obtain the results in Pérez and Yamazaki (2016). When the positive jumps of the Lévy process are hyper-exponential compound Poisson distributed, the first suboptimal problem becomes that in Avanzi et al. (2014). If the dividend decision intensity goes to infinity, and meanwhile the fixed costs on capital injection tend to zero, the two suboptimal problems in this paper reduce to those in Bayraktar et al. (2013). Furthermore, for 
hyper-exponential compound Poisson positive jumps, our results are consistent with Avanzi et al. (2011).

This paper is organized as follows. Section 2 provides the formulations of the problem. Section 3 discusses the case without capital injection. The case with incorporated capital injection is considered in Section 4. The optimal periodic dividend and capital injection strategies are derived in Section 5. Section 6 gives some numerical examples.

\section{Model and Optimal Control Problem}

\subsection{Spectrally Positive Lévy Processes}

Let $X=\left\{X_{t}\right\}$ be a spectrally positive Lévy process with non-monotone paths on a filtered probability space $(\Omega, \mathcal{F}, \mathbb{F}, \mathbb{P})$, where $\mathbb{F}=\left\{\mathcal{F}_{t}\right\}$ satisfies the usual conditions. The Lévy triplet of $X$ is given by $(c, \sigma, \nu)$, where $c>0, \sigma \geq 0$, and $\nu$ is a Lévy measure on $(0, \infty)$ satisfying the integrability condition $\int_{0}^{\infty}\left(1 \wedge x^{2}\right) \nu(d x)<\infty$. Let $E^{x}$ be the conditional expectation given the initial surplus $x$, and in particular, denote $E^{0}$ by $E$. The Laplace exponent of $X$ is given by

$$
\psi(s)=\frac{1}{t} \log E\left[e^{-s X_{t}}\right]=\frac{\sigma^{2}}{2} s^{2}+c s+\int_{0}^{\infty}\left(e^{-s x}-1+s x \mathbf{1}_{\{0<x \leq 1\}}\right) \nu(d x),
$$

where $\mathbf{1}_{A}$ is an indicator function of a set $A$. It is well known that $\psi(s)$ is strictly convex and tends to infinity as $s$ tends to infinity. This allows us to define for $q \geq 0$,

$$
\Phi(q)=\sup \{s \geq 0: \psi(s)=q\}
$$

which refers to the largest root of the equation $\psi(s)=q$. Note that the Laplace exponent $\psi$ in (2.1) is known to be zero at the origin, and hence $\Phi(q)$ is strictly positive for $q>0$. The process $X$ has paths of bounded variation if and only if $\sigma=0$ and $\int_{0}^{1} x \nu(d x)<\infty$. Correspondingly, the Laplace exponent (2.1) can be written as

$$
\psi(s)=c_{0} s+\int_{0}^{\infty}\left(e^{-s x}-1\right) \nu(d x),
$$

where $c_{0}=c+\int_{0}^{1} x \nu(d x)$. We rule out the case that $X$ has monotone paths, and then $c_{0}>0$ is necessary when $X$ is of bounded variation. The drift of $X$ is given by

$$
\mu=E\left[X_{1}\right]=-\psi^{\prime}(0+) .
$$

It is well known that if $\int_{1}^{\infty} y \nu(d y)<\infty$, then $\mu=-c+\int_{1}^{\infty} y \nu(d y)<\infty$. In this paper, we assume $\mu<\infty$ to ensure that the optimal problem has a nontrivial solution. For more details on Lévy processes, the reader is referred to Bertoin (1996), Kyprianou (2006) and Kuznetsov et al. (2012). 


\subsection{Formulations of Control Problem}

We assume that the surplus process of a company is modeled by the Lévy process $X$, whose Laplace exponent is given by (2.1) and $X_{0}=x \geq 0$. A control strategy composes of two parts: dividend payment and capital injection. We assume that the dividend payments follow a periodic dividend strategy, where the dividend decision times are governed by a $\mathbb{F}$-adapted and independent Poisson process $N=\left\{N_{t}\right\}$ with intensity $\gamma>0$. Then the dividend payment process $L=\left\{L_{t}\right\}$ is given by

$$
L_{t}=\int_{0}^{t} \vartheta_{s} d N_{s}=\sum_{k=1}^{\infty} \vartheta_{T_{k}} \mathbf{1}_{\left\{T_{k} \leq t\right\}}, \quad t \geq 0,
$$

where $\vartheta_{t} \geq 0$ is the dividend payment at time $t$, and $T_{k}$ is the time of $k$ th dividend. The capital injection process $G=\left\{G_{t}=\sum_{n=1}^{\infty} \mathbf{1}_{\left\{\tau_{n} \leq t\right\}} \xi_{n}\right\}$ is described by a sequence of increasing stopping times $\left\{\tau_{n}, n=1,2, \cdots\right\}$ and a sequence of random variables $\left\{\xi_{n}, n=1,2, \cdots\right\}$, which correspond to the timings and the amounts of capital injection, respectively.

Given a control strategy $\pi=\left(L^{\pi} ; G^{\pi}\right)=\left(\vartheta_{T_{1}}^{\pi}, \vartheta_{T_{2}}^{\pi}, \cdots ; \tau_{1}^{\pi}, \tau_{2}^{\pi}, \cdots ; \xi_{1}^{\pi}, \xi_{2}^{\pi}, \cdots\right)$, the dynamics of the resulting surplus process $X^{\pi}=\left\{X_{t}^{\pi}\right\}$ can be written as

$$
X_{t}^{\pi}=X_{t}-\sum_{k=1}^{\infty} \vartheta_{T_{k}}^{\pi} \mathbf{1}_{\left\{T_{k} \leq t\right\}}+\sum_{n=1}^{\infty} \xi_{n}^{\pi} \mathbf{1}_{\left\{\tau_{n}^{\pi} \leq t\right\}}, \quad t \geq 0 .
$$

Definition 2.1. A strategy $\pi$ is said to be admissible iff

(i) $\left\{L_{t}^{\pi}\right\}_{t \geq 0}$ is an increasing and $\mathbb{F}$-adapted càdlàg process satisfying $L_{0}=0$,

$L_{t}^{\pi}=\int_{0}^{t} \vartheta_{s}^{\pi} d N_{s}$ and $\Delta L_{T_{j}}^{\pi}=\vartheta_{T_{j}}^{\pi} \leq X_{T_{j}-}^{\pi}+\Delta X_{T_{j}}$ for $j=1,2, \cdots$;

(ii) $\tau_{n}^{\pi}$ is a stopping time with respect to $\mathbb{F}$, and $0 \leq \tau_{1}^{\pi}<\tau_{2}^{\pi}<\cdots$ a.s.;

(iii) $\xi_{n}^{\pi}$ is nonnegative and measurable with respect to $\mathcal{F}_{\tau_{n}^{\pi}}, n=1,2, \cdots$;

(iv) $P\left(\lim _{n \rightarrow \infty} \tau_{n}^{\pi} \leq a\right)=0, \forall a \geq 0$.

Let $\Pi$ denote the set of admissible strategies. Define the time of ruin by

$$
T^{\pi}=\inf \left\{t \geq 0: X_{t}^{\pi} \leq 0\right\},
$$

with $\inf \emptyset=\infty$ by convention. To incorporate the capital injection, the fixed and proportional transaction costs are considered. When the amount of capital is $\xi$, we assume that $(\phi-1) \xi$ with $\phi>1$ is the proportional cost, and that $K>0$ is the fixed cost. The time preference of investors is described by the force of interest $\delta>0$. Given a strategy $\pi \in \Pi$, the performance function is defined by

$$
V(x ; \pi)=E^{x}\left[\int_{0}^{T^{\pi}} e^{-\delta s} \vartheta_{s}^{\pi} d N_{s}-\sum_{n=1}^{\infty} e^{-\delta \tau_{n}^{\pi}}\left(K+\phi \xi_{n}^{\pi}\right) \mathbf{1}_{\left\{\tau_{n}^{\pi} \leq T^{\pi}\right\}}\right] .
$$


Our objective is to find the optimal return function or the value function, defined as

$$
V(x)=\sup _{\pi \in \Pi} V(x ; \pi),
$$

and the associated optimal strategy $\pi^{*} \in \Pi$ such that $V(x)=V\left(x ; \pi^{*}\right)$.

\subsection{Preliminary Discussions for Optimal Problem}

By the optimality of the value function $V(x)$ and similar to Lemma 3.1 in Peng et al. (2012), we obtain that $V(x)$ is increasing and satisfies

$$
0 \leq V(0) \leq V(x) ; \quad 0 \leq V(x)-V(y) \leq \phi(x-y)+K, 0 \leq y \leq x .
$$

To derive the optimal strategy and the value function, the Quasi-Variational-Inequality (see, e.g., Fleming and Soner (2006)) approach is adopted. Before that, we introduce some operators. Throughout the paper, for a set $D \subset \mathbb{R}$, a function $p: D \rightarrow \mathbb{R}$ is called sufficiently smooth meaning that it belongs to $\mathcal{C}^{1}(D)$ if $X$ is of bounded variation, otherwise it belongs to $\mathcal{C}^{2}(D)$. Suppose that a sufficiently smooth function $v$ is a candidate function for the value function. Let $\mathscr{A}$ denote the extended generator of the process $X$, defined by

$$
\mathscr{A} v(x)=\frac{\sigma^{2}}{2} v^{\prime \prime}(x)-c v^{\prime}(x)+\int_{0}^{\infty}\left[v(x+y)-v(x)-v^{\prime}(x) y \mathbf{1}_{\{0<y \leq 1\}}\right] \nu(d y) .
$$

Similar to Avanzi et al. (2014), we give the operator $\mathcal{N}$ associated with the dividends by

$$
\mathcal{N} v(x)=\gamma \max _{0 \leq l \leq x}\{l+v(x-l)-v(x)\} .
$$

Let $\mathcal{M}$ denote the injection operator, defined by

$$
\mathcal{M} v(x)=\sup _{y \geq 0}\{v(x+y)-K-\phi y\}
$$

Due to the time value of money, we give the following lemma whose proof is given in Appendix A.

Lemma 2.1. It is optimal to postpone the capital injection as long as possible, i.e., if capital injection occurs, it happens only at the moment when the reserve process hits the barrier 0 .

Theorem 2.1. Let $v(x)$ be an increasing and sufficiently smooth function on $(0, \infty)$ satisfying

$$
\max \{\mathcal{N} v(x)+(\mathscr{A}-\delta) v(x), \mathcal{M} v(x)-v(x)\} \leq 0, \quad x>0
$$

with the initial conditions $v(0) \geq 0$ and $\mathcal{M} v(0) \leq v(0)$. Then we have $v(x) \geq V(x)$. 
Proof. By the definition of the performance function and Lemma 2.1, and noting that the Lévy process $X$ has only positive jumps, we only need to consider the strategies, where the capital injection does not occur at the same time as a jump of either the process $X$ or the dividend process $L$. Under this consideration, for a strategy $\pi \in \Pi$, we can characterize the discontinuous points of the controlled reserve process by the following sets

$$
\begin{aligned}
\Lambda_{t} & =\left\{s \leq t: \Delta X_{s} \neq 0, \Delta L_{s}^{\pi}=0, \Delta G_{s}^{\pi}=0\right\} \\
\Lambda_{t}^{\prime} & =\left\{s \leq t: \Delta L_{s}^{\pi} \neq 0, \Delta G_{s}^{\pi}=0\right\} \\
\Lambda_{t}^{\prime \prime} & =\left\{s \leq t: \Delta G_{s}^{\pi} \neq 0, \Delta L_{s}^{\pi}=0, \Delta X_{s}=0\right\}=\left\{\tau_{i}^{\pi}: \tau_{i}^{\pi} \leq t, i=1,2, \cdots\right\} .
\end{aligned}
$$

Let $\left\{S_{m}\right\}$ be a sequence of stopping times defined by $S_{m}=\inf \left\{t \geq 0: X_{t}^{\pi}>\right.$ $m$ or $\left.X_{t}^{\pi}<\frac{1}{m}\right\}$. Noting the sufficient smoothness of $v$, and applying the Itô's formula for semimartingale, we obtain

$$
\begin{aligned}
& e^{-\delta\left(t \wedge S_{m}\right)} v\left(X_{t \wedge S_{m}}^{\pi}\right) \\
= & v(x)+\int_{0}^{t \wedge S_{m}} e^{-\delta s}\left[\frac{\sigma^{2}}{2} v^{\prime \prime}\left(X_{s-}^{\pi}\right)-\delta v\left(X_{s-}^{\pi}\right)\right] d s+\int_{0}^{t \wedge S_{m}} e^{-\delta s} v^{\prime}\left(X_{s-}^{\pi}\right) d X_{s}^{\pi} \\
& +\sum_{s \in \bar{\Lambda}_{t \wedge S_{m}}} e^{-\delta s}\left[v\left(X_{s}^{\pi}\right)-v\left(X_{s-}^{\pi}\right)-v^{\prime}\left(X_{s-}^{\pi}\right) \Delta X_{s}^{\pi}\right],
\end{aligned}
$$

where $\bar{\Lambda}_{t}=\Lambda_{t} \cup \Lambda_{t}^{\prime} \cup \Lambda_{t}^{\prime \prime}$. By $X_{s}^{\pi}=X_{s}-L_{s}^{\pi}+G_{s}^{\pi}$, we have

$$
\begin{aligned}
& e^{-\delta\left(t \wedge S_{m}\right)} v\left(X_{t \wedge S_{m}}^{\pi}\right) \\
= & v(x)+\int_{0}^{t \wedge S_{m}} e^{-\delta s}\left[\frac{\sigma^{2}}{2} v^{\prime \prime}\left(X_{s-}^{\pi}\right)-\delta v\left(X_{s-}^{\pi}\right)\right] d s+\int_{0}^{t \wedge S_{m}} e^{-\delta s} v^{\prime}\left(X_{s-}^{\pi}\right) d X_{s} \\
& +\sum_{s \in \Lambda_{t \wedge S_{m}}^{\prime}} e^{-\delta s}\left[v\left(X_{s-}^{\pi}+\Delta X_{s}-\vartheta_{s}^{\pi}\right)-v\left(X_{s-}^{\pi}+\Delta X_{s}\right)\right]+\sum_{\tau_{n}^{\pi} \leq t \wedge S_{m}} e^{-\delta \tau_{n}^{\pi}}\left[v\left(\xi_{n}^{\pi}\right)-v(0)\right] \\
& +\sum_{s \in \Lambda_{t \wedge S_{m}}} e^{-\delta s}\left[v\left(X_{s-}^{\pi}+\Delta X_{s}\right)-v\left(X_{s-}^{\pi}\right)-v^{\prime}\left(X_{s-}^{\pi}\right) \Delta X_{s}\right] .
\end{aligned}
$$

Note

$$
\begin{aligned}
\sum_{s \in \Lambda_{t \wedge S_{m}}} e^{-\delta s} v^{\prime}\left(X_{s-}^{\pi}\right) \Delta X_{s}= & \sum_{s \in \Lambda_{t \wedge S_{m}}} e^{-\delta s} v^{\prime}\left(X_{s-}^{\pi}\right) \Delta X_{s} \mathbf{1}_{\left\{\left|\Delta X_{s}\right| \leq 1\right\}} \\
& +\int_{0}^{t \wedge S_{m}} e^{-\delta s} v^{\prime}\left(X_{s-}^{\pi}\right) d\left(\sum_{u \in \Lambda_{s}} \Delta X_{u} \mathbf{1}_{\left\{\left|\Delta X_{u}\right|>1\right\}}\right)
\end{aligned}
$$


and

$$
\begin{aligned}
& \sum_{s \in \Lambda_{t \wedge S_{m}}^{\prime}} e^{-\delta s}\left[v\left(X_{s-}^{\pi}+\Delta X_{s}-\vartheta_{s}^{\pi}\right)-v\left(X_{s-}^{\pi}+\Delta X_{s}\right)\right] \\
= & \int_{0}^{t \wedge S_{m}} e^{-\delta s}\left[v\left(X_{s-}^{\pi}+\Delta X_{s}-\vartheta_{s}^{\pi}\right)-v\left(X_{s-}^{\pi}+\Delta X_{s}\right)\right] d N_{s} .
\end{aligned}
$$

We obtain

$$
\begin{aligned}
e^{-\delta\left(t \wedge S_{m}\right)} v\left(X_{t \wedge S_{m}}^{\pi}\right)= & v(x)+\widetilde{M}_{t \wedge S_{m}}+\sum_{i=1}^{3} M_{t \wedge S_{m}}^{i} \\
& -\int_{0}^{t \wedge S_{m}} e^{-\delta s} \vartheta_{s}^{\pi} d N_{s}+\sum_{\tau_{n}^{\pi} \leq t \wedge S_{m}} e^{-\delta \tau_{n}^{\pi}}\left[v\left(\xi_{n}^{\pi}\right)-v(0)\right],
\end{aligned}
$$

where

$$
\begin{aligned}
\widetilde{M}_{t}= & \int_{0}^{t} e^{-\delta s}\left\{(\mathscr{A}-\delta) v\left(X_{s-}^{\pi}\right)+\gamma\left[\vartheta_{s}^{\pi}+v\left(X_{s-}^{\pi}+\Delta X_{s}-\vartheta_{s}^{\pi}\right)-v\left(X_{s-}^{\pi}+\Delta X_{s}\right)\right]\right\} d s, \\
M_{t}^{1}= & \int_{0}^{t} e^{-\delta s} v^{\prime}\left(X_{s-}^{\pi}\right) d\left(X_{s}+c s-\sum_{u \in \Lambda_{s}} \Delta X_{u} \mathbf{1}_{\left\{\left|\Delta X_{u}\right|>1\right\}}\right), \\
M_{t}^{2}= & \sum_{s \in \Lambda_{t}} e^{-\delta s}\left[v\left(X_{s}^{\pi}\right)-v\left(X_{s-}^{\pi}\right)-v^{\prime}\left(X_{s-}^{\pi}\right) \Delta X_{s} \mathbf{1}_{\left\{\left|\Delta X_{s}\right| \leq 1\right\}}\right] \\
& -\int_{0}^{t} \int_{0}^{\infty} e^{-\delta s}\left[v\left(X_{s-}^{\pi}+y\right)-v\left(X_{s-}^{\pi}\right)-v^{\prime}\left(X_{s-}^{\pi}\right) y \mathbf{1}_{\{|y| \leq 1\}}\right] \nu(d y) d s, \\
M_{t}^{3}= & \int_{0}^{t} e^{-\delta s}\left[\vartheta_{s}^{\pi}+v\left(X_{s-}^{\pi}+\Delta X_{s}-\vartheta_{s}^{\pi}\right)-v\left(X_{s-}^{\pi}+\Delta X_{s}\right)\right]\left(d N_{s}-\gamma d s\right) .
\end{aligned}
$$

By the Lévy-Itô decomposition, $\left\{M_{t \wedge S_{m}}^{1}\right\}$ is a zero-mean martingale; and by the compensation formula, $\left\{M_{t \wedge S_{m}}^{i}\right\}$ for $i=2,3$ are zero-mean martingales. Taking expectations on both sides of (2.11) and noting $(\mathscr{A}-\delta) v(x)+\gamma[l+v(x-l)-v(x) \leq 0$ for $0 \leq l \leq x, v(x)-v(0) \leq \phi x+K$ and $v(x) \geq 0$, we obtain

$$
v(x) \geq E^{x}\left[\int_{0}^{t \wedge S_{m}} e^{-\delta s} \vartheta_{s}^{\pi} d N_{s}-\sum_{n=1}^{\infty} e^{-\delta \tau_{n}^{\pi}}\left(K+\phi \xi_{n}^{\pi}\right) \mathbf{1}_{\left\{\tau_{n}^{\pi} \leq t \wedge S_{m}\right\}}\right]
$$

Letting $t$ and $m$ go to infinity, noting $S_{m} \rightarrow T^{\pi}(m \rightarrow \infty)$ a.s., and using Fatou's lemma, we get $v(x) \geq V(x ; \pi)$ for any $\pi \in \Pi$, and so $v(x) \geq V(x)$. 


\section{Optimal Control Problem without Capital Injection}

\subsection{Preliminary Discussions for the Optimal Problem without Capital Injection}

We now study the optimal problem without capital injection. Let $\Pi_{p}=\left\{\pi_{p}\right.$ : $\left.\pi_{p}=\left(L^{\pi_{p}} ; 0\right) \in \Pi\right\} \subset \Pi$ denote the set of all admissible strategies for this suboptimal problem. The value function $V_{p}(x)$ is defined by

$$
V_{p}(x)=\sup _{\pi_{p} \in \Pi_{p}} V\left(x ; \pi_{p}\right)=\sup _{\pi_{p} \in \Pi_{p}} E^{x}\left[\int_{0}^{T^{\pi_{p}}} e^{-\delta s} \vartheta_{s}^{\pi_{p}} d N_{s}\right] .
$$

The objective is to find the optimal strategy $\pi_{p}^{*} \in \Pi_{p}$ such that $V_{p}(x)=V\left(x ; \pi_{p}^{*}\right)$.

By the definition of $V_{p}(x)$, we know $V_{p}(x)$ is an increasing function with $V_{p}(0)=0$. Similar to Theorem 2.1, we give the following lemma.

Lemma 3.1. Let $v_{p}(x)$ be an increasing and sufficiently smooth function on $(0, \infty)$ satisfying

$$
\mathcal{N} v_{p}(x)+(\mathscr{A}-\delta) v_{p}(x) \leq 0, \quad x>0
$$

with the initial conditions $v_{p}(0)=0$. Then we have $v_{p}(x) \geq V_{p}(x)$.

Lemma 3.2. Assuming that $v_{p}(x)$ is an increasing and sufficiently smooth function on $(0, \infty)$ with $v_{p}(0)=0$, and that one of the following two hypotheses holds, we have $v_{p}(x) \geq V_{p}(x)$.

(i) If $v_{p}(x)$ is concave and there exists $x_{p}^{*}>0$ such that $v_{p}^{\prime}\left(x_{p}^{*}\right)=1$ and

$$
\begin{aligned}
& (\mathscr{A}-\delta) v_{p}(x)=0, \quad 0<x<x_{p}^{*}, \\
& (\mathscr{A}-\delta) v_{p}(x)+\gamma\left[x-x_{p}^{*}+v_{p}\left(x_{p}^{*}\right)-v_{p}(x)\right]=0, \quad x \geq x_{p}^{*} .
\end{aligned}
$$

(ii) If for all $x>0, v_{p}^{\prime}(x) \leq 1$ and

$$
(\mathscr{A}-\delta) v_{p}(x)+\gamma\left[x-v_{p}(x)\right]=0 .
$$

Proof. By Lemma 3.1, we only need to prove that the function $v_{p}(x)$ satisfies the inequality in (3.2).

If the hypothesis $(i)$ holds, by the concavity of $v_{p}$, we have

$$
v_{p}^{\prime}(x) \geq 1,0<x<x_{p}^{*} ; \quad v_{p}^{\prime}(x) \leq 1, x \geq x_{p}^{*} .
$$

Then

$$
\max _{0 \leq l \leq x}\left\{l+v_{p}(x-l)\right\}=\left\{\begin{array}{l}
v_{p}(x), \quad 0<x<x_{p}^{*} ; \\
x-x_{p}^{*}+v_{p}\left(x_{p}^{*}\right), \quad x \geq x_{p}^{*} .
\end{array}\right.
$$

Combing (3.3) and (3.4), we obtain that $v_{p}(x)$ satisfies (3.2). 

have

If the hypothesis $(i i)$ holds, we know $\max _{0 \leq l \leq x}\left\{l+v_{p}(x-l)\right\}=x$. By (3.5), we

$$
\gamma \max _{0 \leq l \leq x}\left[l+v_{p}(x-l)-v_{p}(x)\right]+(\mathscr{A}-\delta) v_{p}(x)=0 .
$$

The proof is completed.

Inspired by Avanzi et al. (2014) and the above lemma, we conjecture that the optimal periodic strategy in our setting is likely to be a periodic barrier strategy as defined in below.

Definition 3.1. (Periodic Barrier Dividend Strategy). Under a periodic barrier $x_{p}$, dividend payments are

$$
\vartheta_{T_{i}}^{\pi_{p}}=\max \left(X_{T_{i}-}^{\pi_{p}}+\Delta X_{T_{i}}-x_{p}, 0\right), i=1,2, \cdots,
$$

at all dividend decision times $T_{i}$ before ruin, i.e.,

$$
L_{t}^{\pi_{p}}=\int_{0}^{t}\left(X_{s-}^{\pi_{p}}+\Delta X_{s}-x_{p}\right) \mathbf{1}_{\left\{X_{s-}^{\pi_{p}}+\Delta X_{s} \geq x_{p}\right\}} d N_{s}, \quad t \leq T^{\pi_{p}} .
$$

Under a periodic barrier dividend strategy $\pi_{p}$ with barrier $x_{p}$, the performance function is

$$
V\left(x ; \pi_{p}\right)=E^{x}\left[\int_{0}^{T^{\pi_{p}}} e^{-\delta s}\left(X_{s-}^{\pi_{p}}+\Delta X_{s}-x_{p}\right) \mathbf{1}_{\left\{X_{s-}^{\pi_{p}}+\Delta X_{s} \geq x_{p}\right\}} d N_{s}\right] .
$$

Then the above performance function $V\left(x ; \pi_{p}\right)$ is increasing and $V\left(0 ; \pi_{p}\right)=0$.

Lemma 3.3. Assume that the performance function $V\left(x ; \pi_{p}\right)$ in (3.7) is bounded above by a linear function, sufficiently smooth on $(0, \infty)$ and right continuous at 0 . Then $V\left(x ; \pi_{p}\right)$ satisfies the following integro-differential equations

$$
\begin{aligned}
& (\mathscr{A}-\delta) V\left(x ; \pi_{p}\right)=0, \quad 0<x<x_{p} \\
& (\mathscr{A}-\delta) V\left(x ; \pi_{p}\right)+\gamma\left[x-x_{p}+V\left(x_{p} ; \pi_{p}\right)-V\left(x ; \pi_{p}\right)\right]=0, \quad x \geq x_{p}
\end{aligned}
$$

with the initial value $V\left(0 ; \pi_{p}\right)=0$ and the continuous condition

$$
V\left(x_{p}-; \pi_{p}\right)=V\left(x_{p}+; \pi_{p}\right)=V\left(x_{p} ; \pi_{p}\right)
$$

The corresponding proof is presented in Appendix A. In addition, by Lemma 3.3 in Avanzi et al. (2014), it is known that the ruin is certain under a periodic barrier strategy. 


\subsection{Expression of $V\left(x ; \pi_{p}\right)$ Associated with Periodic Barrier Strategy}

We first discuss the case of $x \geq x_{p}$. Let

$$
\begin{aligned}
& Y_{t}=x_{p}-X_{t}, \quad t \geq 0, \\
& S_{x_{p}}^{-}=\inf \left\{t \geq 0: X_{t}<x_{p}\right\}=\inf \left\{t \geq 0: Y_{t}>0\right\} \triangleq \kappa_{0}^{+}, \\
& r_{0}=\Phi(\delta+\gamma)=\sup \{s \geq 0: \psi(s)=\delta+\gamma\} .
\end{aligned}
$$

Then $\left\{Y_{t}\right\}$ is a spectrally negative Lévy process with the characteristic exponent $\psi(s)$ in $(2.1)$ and $Y_{0}=x_{p}-x$. To find the expression of $V\left(x ; \pi_{p}\right)$, we introduce the following lemma.

Lemma 3.4. For $x \geq x_{p}$, we have

$$
E^{x}\left[e^{-\delta T_{1}}\left(X_{T_{1}}-x_{p}\right) \mathbf{1}_{\left\{T_{1}<S_{x_{p}}^{-}\right\}}\right]=\frac{\gamma}{\delta+\gamma}\left[x-x_{p}+\frac{\mu}{\delta+\gamma}\left(1-e^{-r_{0}\left(x-x_{p}\right)}\right)\right] .
$$

Proof. By (30) in Albrecher et al. (2016), we know, for $z, s \leq 0$,

$$
E^{z}\left[e^{s Y_{e_{q}}} \mathbf{1}_{\left\{e_{q}<\kappa_{0}^{+}\right\}}\right]=\frac{q}{q-\psi(s)}\left(e^{s z}-e^{\Phi(q) z}\right),
$$

where $e_{q}$ has exponential distribution with rate $q>0$ and is independent of the Lévy process $X$. Differentiating with respect to $s$ and letting $s$ tend to zero in the above equation yield

$$
E^{z}\left[Y_{e_{q}} \mathbf{1}_{\left\{e_{q}<\kappa_{0}^{+}\right\}}\right]=z+\frac{\psi^{\prime}(0+)}{q}\left(1-e^{\Phi(q) z}\right) .
$$

Then, we have

$$
\begin{aligned}
E^{x}\left[e^{-\delta T_{1}}\left(X_{T_{1}}-x_{p}\right) \mathbf{1}_{\left\{T_{1}<S_{x_{p}}^{-}\right\}}\right] & =-E^{x_{p}-x}\left[e^{-\delta T_{1}} Y_{T_{1}} \mathbf{1}_{\left\{T_{1}<\kappa_{0}^{+}\right\}}\right] \\
& =-\frac{\gamma}{\delta+\gamma} E^{x_{p}-x}\left[Y_{e_{(\delta+\gamma)}} \mathbf{1}_{\left\{e_{(\delta+\gamma)}<\kappa_{0}^{+}\right\}}\right],
\end{aligned}
$$

where $e_{(\delta+\gamma)}$ is exponentially distributed with rate $\delta+\gamma$, and the second equality is due to the law of total probability. Noting $\psi^{\prime}(0+)=-\mu$, the result is obtained by substituting (3.15) into (3.16).

Theorem 3.1. For $x \geq x_{p}$, the performance function $V\left(x ; \pi_{p}\right)$ in (3.7) is given by

$$
V\left(x ; \pi_{p}\right)=\frac{\gamma}{\delta+\gamma} A_{1}\left(x-x_{p}\right)+V\left(x_{p} ; \pi_{p}\right) B_{1}\left(x-x_{p}\right),
$$


where

$$
\begin{aligned}
& A_{1}(x)=x+\frac{\mu}{\delta+\gamma}\left(1-e^{-r_{0} x}\right), \\
& B_{1}(x)=\frac{\gamma}{\delta+\gamma}+\frac{\delta}{\delta+\gamma} e^{-r_{0} x}
\end{aligned}
$$

Proof. By the strong Markov property and $X_{S_{x_{p}}^{-}}=x_{p}$ a.s., we have

$$
V\left(x ; \pi_{p}\right)=E^{x}\left[e^{-\delta T_{1}}\left(X_{T_{1}}-x_{p}\right) \mathbf{1}_{\left\{T_{1}<S_{x_{p}}^{-}\right\}}\right]+V\left(x_{p} ; \pi_{p}\right) E^{x}\left[e^{-\delta\left(T_{1} \wedge S_{x_{p}}^{-}\right)}\right] .
$$

By the law of total probability, we obtain

$$
\begin{aligned}
E^{x}\left[e^{-\delta\left(T_{1} \wedge S_{x_{p}}^{-}\right)}\right] & =E^{x}\left[\int_{0}^{\infty} e^{-\delta\left(t \wedge S_{x_{p}}^{-}\right)} \cdot \gamma e^{-\gamma t} d t\right] \\
& =\frac{\gamma}{\delta+\gamma}+\frac{\delta}{\delta+\gamma} E^{x}\left[e^{-(\delta+\gamma) S_{x_{p}}^{-}}\right] \\
& =\frac{\gamma}{\delta+\gamma}+\frac{\delta}{\delta+\gamma} e^{-r_{0}\left(x-x_{p}\right)},
\end{aligned}
$$

where the last equality is due to (19) in Kuznetsov et al. (2012). The result is yielded by substituting (3.14) and (3.21) into (3.20).

Remark 3.1. (i) From (3.17), it is known that, for $x>x_{p}, V\left(x ; \pi_{p}\right)$ is infinitely differentiable and bounded above by a linear function.

(ii) As $\lim _{\gamma \rightarrow \infty} r_{0}=\infty$, we have $\lim _{\gamma \rightarrow \infty} V\left(x ; \pi_{p}\right)=x-x_{p}+V\left(x_{p} ; \pi_{p}\right)$ for $x \geq x_{p}$. Then the dividend payments could occur at any time (continuously) as the limit goes to infinity, the periodic barrier strategy becomes a barrier dividend strategy.

(iii) By (3.17), we have

$$
\begin{aligned}
& V^{\prime}\left(x_{p}+; \pi_{p}\right)=\frac{\gamma}{\delta+\gamma}\left(1+\frac{\mu r_{0}}{\delta+\gamma}\right)-\frac{\delta r_{0}}{\delta+\gamma} V\left(x_{p} ; \pi_{p}\right) ; \\
& V^{\prime \prime}\left(x_{p}+; \pi_{p}\right)=-\frac{\gamma \mu}{(\delta+\gamma)^{2}} r_{0}^{2}+\frac{\delta}{\delta+\gamma} r_{0}^{2} V\left(x_{p} ; \pi_{p}\right) .
\end{aligned}
$$

In the following, we discuss the case of $0<x<x_{p}$. We will used the scale functions $W^{(q)}(x), Z^{(q)}(x), \bar{Z}^{(q)}(x)$ and $Z^{(q)}(x, s)$, which are studied in Kuznetsov et al. (2012) and Albrecher et al. (2016), and whose definitions and some results are shown in Appendix B. For the uncontrolled surplus process $X$, let

$$
\begin{aligned}
& S_{0}^{-}=\inf \left\{t \geq 0: X_{t}<0\right\}=\inf \left\{t \geq 0: Y_{t}>x_{p}\right\} \triangleq \kappa_{x_{p}}^{+} \\
& T_{x_{p}}^{+}=\min \left\{T_{i}: X_{T_{i}}>x_{p}, i=1,2 \cdots\right\}=\min \left\{T_{i}: Y_{T_{i}}<0, i=1,2 \cdots\right\} \triangleq \bar{T}_{0}^{-},
\end{aligned}
$$


where $Y_{t}$ is defined by $(3.11)$.

Lemma 3.5. For $0 \leq x<x_{p}$, we have

$$
\begin{aligned}
& E^{x}\left[e^{-\delta T_{x_{p}}^{+}}\left(X_{T_{x_{p}}^{+}}-x_{p}\right) \mathbf{1}_{\left\{T_{x_{p}}^{+}<S_{0}^{-}\right\}}\right]=I_{1}\left(x_{p}-x, x_{p}\right) ; \\
& E^{x}\left[e^{-\delta T_{x_{p}}^{+}} \mathbf{1}_{\left\{T_{x_{p}}^{+}<S_{0}^{-}\right\}}\right]=I_{2}\left(x_{p}-x, x_{p}\right),
\end{aligned}
$$

where

$$
\begin{aligned}
& I_{1}\left(x, x_{p}\right)=\frac{\gamma}{\delta+\gamma}\left[D(x)-\frac{Z^{(\delta)}\left(x, r_{0}\right)}{Z^{(\delta)}\left(x_{p}, r_{0}\right)} D\left(x_{p}\right)\right], \\
& D(x)=-\frac{\mu \gamma}{\delta(\delta+\gamma)} Z^{(\delta)}(x)-\bar{Z}^{(\delta)}(x)+\frac{\mu}{\delta} \\
& I_{2}\left(x, x_{p}\right)=\frac{\gamma}{\delta+\gamma}\left[Z^{(\delta)}(x)-\frac{Z^{(\delta)}\left(x, r_{0}\right)}{Z^{(\delta)}\left(x_{p}, r_{0}\right)} Z^{(\delta)}\left(x_{p}\right)\right] .
\end{aligned}
$$

Proof. The result is obvious when $x=0$, and then we only need to discuss the case of $0<x<x_{p}$. By (15) in Albrecher et al. (2016), we have, for $0<y<x_{p}$,

$$
E^{y}\left[e^{-\delta \bar{T}_{0}^{-}+s Y_{\bar{T}_{0}^{-}}} \mathbf{1}_{\left\{\bar{T}_{0}^{-}<\kappa_{x_{p}}^{+}\right\}}\right]=\frac{\gamma}{\delta+\gamma-\psi(s)}\left(Z^{(\delta)}(y, s)-\frac{Z^{(\delta)}\left(y, r_{0}\right)}{Z^{(\delta)}\left(x_{p}, r_{0}\right)} Z^{(\delta)}\left(x_{p}, s\right)\right) .
$$

Letting $s$ go to zero and noting

$$
E^{x}\left[e^{-\delta T_{x_{p}}^{+}} \mathbf{1}_{\left\{T_{x_{p}}^{+}<S_{0}^{-}\right\}}\right]=E^{x_{p}-x}\left[e^{-\delta \bar{T}_{0}^{-}} \mathbf{1}_{\left\{\bar{T}_{0}^{-}<\kappa_{x_{p}}^{+}\right\}}\right],
$$

we have the result in (3.24). Differentiating with respect to $s$, letting $s$ tend to zero in (3.28), and noting

$$
\begin{aligned}
& \left.\frac{\partial Z^{(\delta)}(y, s)}{\partial s}\right|_{s=0}=\bar{Z}^{(\delta)}(y)-\frac{\mu}{\delta}+\frac{\mu}{\delta} Z^{(\delta)}(y) \\
& E^{x}\left[e^{-\delta T_{x_{p}}^{+}}\left(X_{T_{x_{p}}^{+}}-b\right) \mathbf{1}_{\left\{T_{x_{p}}^{+}<S_{0}^{-}\right\}}\right]=-E^{x_{p}-x}\left[e^{-\delta \bar{T}_{0}^{-}} Y_{\bar{T}_{0}^{-}} \mathbf{1}_{\left\{\bar{T}_{0}^{-}<\kappa_{x p}^{+}\right\}}\right],
\end{aligned}
$$

we obtain the result in (3.23) after some calculations.

Theorem 3.2. For $0 \leq x<x_{p}$, the performance function $V\left(x ; \pi_{p}\right)$ in (3.7) is given by

$$
V\left(x ; \pi_{p}\right)=\frac{\gamma}{\delta+\gamma} A_{2}\left(x_{p}-x\right)+V\left(x_{p} ; \pi_{p}\right) B_{2}\left(x_{p}-x\right)
$$


where $V\left(x_{p} ; \pi_{p}\right)=\frac{\gamma}{\delta+\gamma}\left[\frac{\bar{Z}^{(\delta)}\left(x_{p}\right)-\frac{\mu}{\delta}}{B_{2}\left(x_{p}\right)}+\frac{\mu}{\delta}\right]$ and

$$
\begin{aligned}
& A_{2}(x)=-\frac{\mu}{\delta} B_{2}(x)-\bar{Z}^{(\delta)}(x)+\frac{\mu}{\delta} \\
& B_{2}(x)=\frac{\delta}{\delta+\gamma} Z^{(\delta)}\left(x, r_{0}\right)+\frac{\gamma}{\delta+\gamma} Z^{(\delta)}(x) .
\end{aligned}
$$

Proof. By the strong Markov property and Lemma 3.5, we have

$$
V\left(x ; \pi_{p}\right)=I_{1}\left(x_{p}-x, x_{p}\right)+V\left(x_{p} ; \pi_{p}\right) I_{2}\left(x_{p}-x, x_{p}\right) .
$$

Letting $x$ tend to $x_{p}$ in the above equation yields

$$
I_{1}\left(0, x_{p}\right)=\left(1-I_{2}\left(0, x_{p}\right)\right) V\left(x_{p} ; \pi_{p}\right) .
$$

By (3.25), we have

$$
\begin{aligned}
& I_{1}\left(x, x_{p}\right)=\frac{\gamma}{\delta+\gamma} D(x)-\frac{\gamma}{\delta+\gamma} Z^{(\delta)}\left(x_{p}, r_{0}\right) \frac{D\left(x_{p}\right)}{Z^{(\delta)}\left(x_{p}, r_{0}\right)} \\
& I_{1}\left(0, x_{p}\right)=\frac{\gamma \mu}{(\delta+\gamma)^{2}}-\frac{\gamma}{\delta+\gamma} \frac{D\left(x_{p}\right)}{Z^{(\delta)}\left(x_{p}, r_{0}\right)}
\end{aligned}
$$

Then

$$
\begin{aligned}
I_{1}\left(x, x_{p}\right) & =\frac{\gamma}{\delta+\gamma} D(x)-Z^{(\delta)}\left(x, r_{0}\right)\left[\frac{\gamma \mu}{(\delta+\gamma)^{2}}-I_{1}\left(0, x_{p}\right)\right] \\
& =\frac{\gamma}{\delta+\gamma} A_{2}(x)+Z^{(\delta)}\left(x, r_{0}\right)\left(1-I_{2}\left(0, x_{p}\right)\right) V\left(x_{p} ; \pi_{p}\right) .
\end{aligned}
$$

Since

$$
I_{2}\left(x, x_{p}\right)=\frac{\gamma}{\delta+\gamma} Z^{(\delta)}(x)-Z^{(\delta)}\left(x, r_{0}\right)\left(\frac{\gamma}{\delta+\gamma}-I_{2}\left(0, x_{p}\right)\right)
$$

we obtain (3.29) by substituting (3.34) and (3.35) into (3.32). Letting $x$ tend to zero and noting $V\left(0 ; \pi_{p}\right)=0$, we get the expression of $V\left(x_{p} ; \pi_{p}\right)$.

Remark 3.2. (i) By the definitions of scale functions in Appendix B, if $x<0$, we have $A_{2}(x)=A_{1}(x)$ and $B_{2}(x)=B_{1}(x)$, and so the the value function can be written uniformly as (3.29) for $x \in \mathbb{R}$. Substituting the value of $V\left(x_{p} ; \pi_{p}\right)$ into (3.29), we obtain

$$
V\left(x ; \pi_{p}\right)=\frac{\gamma}{\delta+\gamma}\left[\frac{B_{2}\left(x_{p}-x\right)}{B_{2}\left(x_{p}\right)} \bar{Z}^{(\delta)}\left(x_{p}\right)-\bar{Z}^{(\delta)}\left(x_{p}-x\right)-\frac{\mu}{\delta}\left(\frac{B_{2}\left(x_{p}-x\right)}{B_{2}\left(x_{p}\right)}-1\right)\right],
$$


which is consistent with (3.15) in Pérez and Yamazaki (2016). If $\mu$ tends to $+\infty$, we have that $r_{0}$ goes to zero, furthermore,

$$
\lim _{\mu \rightarrow+\infty} \frac{B_{2}\left(x_{p}-x\right)}{B_{2}\left(x_{p}\right)}-1=\frac{Z^{(\delta)}\left(x_{p}-x\right)}{Z^{(\delta)}\left(x_{p}\right)}-1<0, \quad(x>0) .
$$

Hence, we obtain $\lim _{\mu \rightarrow+\infty} V\left(x ; \pi_{p}\right)=+\infty$ for any $x_{p}>0$. In other words, the solution of the optimal problem is trivial if the drift $\mu=+\infty$.

(ii) By the definitions of $r_{0}$ in (3.13) and $Z^{(\delta)}(x, s)$ in (B.1), we have

$$
Z^{(\delta)}\left(x, r_{0}\right)=e^{r_{0} x}\left[1-\gamma \int_{0}^{x} W^{(\delta)}(y) d y\right], \quad \frac{\partial Z^{(\delta)}\left(x, r_{0}\right)}{\partial x}=r_{0} Z^{(\delta)}\left(x, r_{0}\right)-\gamma W^{(\delta)}(x) .
$$

Then by (3.29), we have

$$
V^{\prime}\left(x_{p}-; \pi_{p}\right)=\frac{\gamma}{\delta+\gamma}\left(1+\frac{\mu r_{0}}{\delta+\gamma}\right)-\frac{\delta r_{0}}{\delta+\gamma} V\left(x_{p} ; \pi_{p}\right),
$$

and when $X$ is of unbounded variation, by (B.3),

$$
V^{\prime \prime}\left(x_{p}-; \pi_{p}\right)=-\frac{\gamma \mu}{(\delta+\gamma)^{2}} r_{0}^{2}+\frac{\delta}{\delta+\gamma} r_{0}^{2} V\left(x_{p} ; \pi_{p}\right)
$$

Combining (3.22), we obtain that $V\left(x ; \pi_{p}\right)$ is sufficiently smooth on $(0, \infty)$.

\subsection{Optimal Strategy for the Optimal Problem without Capital Injection}

By Lemma 3.2 and Lemma 3.3, if a periodic dividend strategy $\pi_{p}^{*}$ with barrier $x_{p}^{*}>0$ is optimal, we have $V^{\prime}\left(x_{p}^{*} ; \pi_{p}^{*}\right)=1$. Furthermore, by (3.36) we get

$$
V\left(x_{p}^{*} ; \pi_{p}^{*}\right)=\frac{\gamma}{\delta+\gamma} \frac{\mu}{\delta}-\frac{1}{r_{0}} .
$$

Substituting the above equation into (3.17) and (3.29), we obtain

$$
V\left(x ; \pi_{p}^{*}\right)=\left\{\begin{array}{l}
-\frac{\gamma \bar{Z}^{(\delta)}\left(x_{p}^{*}-x\right)}{\gamma+\delta}-\frac{R\left(x_{p}^{*}-x\right)}{(\gamma+\delta) r_{0}}+\frac{\gamma}{\gamma+\delta} \frac{\mu}{\delta}, 0 \leq x<x_{p}^{*}, \\
-\frac{\delta}{(\gamma+\delta) r_{0}} e^{-r_{0}\left(x-x_{p}^{*}\right)}+\frac{\gamma}{\gamma+\delta}\left(x-x_{p}^{*}-\frac{1}{r_{0}}+\frac{\mu}{\delta}\right), x \geq x_{p}^{*},
\end{array}\right.
$$

where

$$
R(x)=\delta Z^{(\delta)}\left(x, r_{0}\right)+\gamma Z^{(\delta)}(x),
$$


and $x_{p}^{*}$ is determined by $V\left(0 ; x_{p}^{*}\right)=0$, i.e.,

$$
\frac{\gamma \bar{Z}^{(\delta)}\left(x_{p}^{*}\right)}{\gamma+\delta}+\frac{R\left(x_{p}^{*}\right)}{(\gamma+\delta) r_{0}}-\frac{\gamma}{\gamma+\delta} \frac{\mu}{\delta}=0 .
$$

The following lemma, which is Lemma 4.2 in Pérez and Yamazaki (2016), discusses the solution of the above equation.

Lemma 3.6. The equation (3.40) has a unique positive root $x_{p}^{*}>0$ if and only if $\frac{\gamma}{\gamma+\delta} \frac{\mu}{\delta}-\frac{1}{r_{0}}>0$.

Remark 3.3. (i) There exists a solution $x_{p}^{*}>0$ of (3.40) if and only if $V\left(x_{p}^{*} ; \pi_{p}^{*}\right)>0$ by (3.37). This follows from the increasing property of $V\left(x ; \pi_{p}^{*}\right)$ and $V\left(0 ; x_{p}^{*}\right)=0$.

(ii) Similar to Remark 4.5 in Avanzi et al. (2014), we illustrate Lemma 3.6.

In the view of the dividend frequency, the inequality $\frac{\gamma}{\gamma+\delta} \frac{\mu}{\delta}-\frac{1}{r_{0}}>0$ can be rewritten as $\gamma>\gamma_{0}$, where $\gamma_{0}$ is the solution to $\frac{\gamma_{0}}{\gamma_{0}+\delta} \frac{\mu}{\delta}-\frac{1}{r_{0}\left(\gamma_{0}\right)}=0$. In a sense, larger rate of dividend results in higher barrier of dividend.

From the perspective of the overall drift of $X$, the inequality $\frac{\gamma}{\gamma+\delta} \frac{\mu}{\delta}-\frac{1}{r_{0}}>0$ can be rewritten as $\mu>\mu_{0}$, where $\mu_{0}$ is the solution to $\frac{\gamma}{\gamma+\delta} \frac{\mu_{0}}{\delta}-\frac{1}{r_{0}}=0$. Then $\mu_{0}>0$, and $\lim _{\gamma \rightarrow \infty} \mu_{0}=0$ which is the condition of (2.13) in Bayraktar et al. (2013).

Theorem 3.3. (i) If $\frac{\gamma}{\gamma+\delta} \frac{\mu}{\delta}-\frac{1}{r_{0}} \leq 0$, we have $V_{p}(x)=V\left(x ; \pi_{p}^{*}\right)$, where

$$
V\left(x ; \pi_{p}^{*}\right)=-\frac{\gamma \mu}{(\gamma+\delta)^{2}} e^{-r_{0} x}+\frac{\gamma}{\gamma+\delta} x+\frac{\gamma \mu}{(\gamma+\delta)^{2}}, \quad x \geq 0
$$

and the optimal periodic dividend strategy $\pi_{p}^{*}$ is given in (3.6) with $x_{p}^{*}=0$.

(ii) If $\frac{\gamma}{\gamma+\delta} \frac{\mu}{\delta}-\frac{1}{r_{0}}>0$, we have $V_{p}(x)=V\left(x ; \pi_{p}^{*}\right)$, where $V\left(x ; \pi_{p}^{*}\right)$ is given by (3.38), and the optimal periodic dividend strategy $\pi_{p}^{*}$ is given in (3.6) with $x_{p}^{*}>0$ determined by (3.40).

Proof. ( $i$ ) Assume that $\pi_{p}^{*}$ is given by (3.6) with $x_{p}^{*}=0$. By Theorem 3.1, we obtain (3.41). Similar to Lemma 4.4 (ii) in Pérez and Yamazaki (2016), we have $0<V^{\prime}\left(x ; \pi_{p}^{*}\right) \leq 1$ for all $x>0$. The results in $(i)$ follows Lemma 3.3 and $(i i)$ in Lemma 3.2.

(ii) In the case of $\frac{\gamma}{\gamma+\delta} \frac{\mu}{\delta}-\frac{1}{r_{0}}>0$, the equation (3.40) has a unique positive root $x_{p}^{*}$. Lemma $4.4(i)$ in Pérez and Yamazaki (2016) shows that $V\left(x ; \pi_{p}^{*}\right)$ in $(3.38)$ is increasing, concave and $V^{\prime}\left(x_{p}^{*} ; \pi_{p}^{*}\right)=1$. By Lemma 3.3, Lemma $3.2(i)$ and Remark $3.2(i i)$, we obtain the results in $(i i)$

Remark 3.4. (i) By the different method, Theorem 3.3 shows the same results in Theorem 4.1 of Pérez and Yamazaki (2016). If $\mu<0$ (including $-\infty$ ), we obtain that the optimal barrier $x_{p}^{*}$ is zero, and that the corresponding strategy is called 
taking all the money and run at the first Poissonian dividend-decision time which is introduced in Pérez and Yamazaki (2016).

(ii) For $R(x)$ in (3.39), we have $\lim _{\gamma \rightarrow \infty} \frac{R\left(x_{p}^{*}-x\right)}{(\gamma+\delta) r_{0}}=0$ by (B.2). Letting $\gamma$ tend to $\infty$ in (3.38), we obtain

$$
\lim _{\gamma \rightarrow \infty} V\left(x ; \pi_{p}^{*}\right)=\left\{\begin{array}{l}
-\bar{Z}^{(\delta)}\left(\tilde{x}_{p}^{*}-x\right)+\frac{\mu}{\delta}, \quad 0 \leq x<\tilde{x}_{p}^{*} \\
x-\tilde{x}_{p}^{*}+\frac{\mu}{\delta}, \quad x \geq \tilde{x}_{p}^{*}
\end{array}\right.
$$

where $\tilde{x}_{p}^{*}$ is determined by $\bar{Z}^{(\delta)}\left(\tilde{x}_{p}^{*}\right)=\frac{\mu}{\delta}$. Therefore, Theorem 3.3 becomes Theorem 2.1 in Bayraktar et al. (2013).

\section{Optimal Control Problem with Capital Injection to Prevent Ruin}

\subsection{Preliminary Discussions for the Optimal Problem without Ruin}

We assume that the company survives forever by forced capital injections. Let $\Pi_{q}$ denote the set of admissible strategies of this suboptimal problem, i.e.,

$$
\Pi_{q}=\left\{\pi_{q}=\left(L^{\pi_{q}} ; G^{\pi_{q}}\right): \pi_{q} \in \Pi \text { such that } X_{t}^{\pi_{q}}>0 \text { for all } t \geq 0\right\} \subset \Pi .
$$

The value function $V_{q}(x)$ is defined by

$$
\begin{aligned}
V_{q}(x) & =\sup _{\pi_{q} \in \Pi_{q}} V\left(x ; \pi_{q}\right) \\
& =\sup _{\pi_{q} \in \Pi_{q}} E^{x}\left[\int_{0}^{\infty} e^{-\delta s} \vartheta_{s}^{\pi_{q}} d N_{s}-\sum_{n=1}^{\infty} e^{-\delta \tau_{n}^{\pi_{q}}}\left(K+\phi \xi_{n}^{\pi_{q}}\right)\right] .
\end{aligned}
$$

We will search for the optimal strategy $\pi_{q}^{*} \in \Pi_{q}$ and the associated value function $V_{q}(x)=V\left(x ; \pi_{q}^{*}\right)$. By the similar proof of Theorem 2.1, we give the following lemma.

Lemma 4.1. Let $v_{q}(x)$ be an increasing and sufficiently smooth function on $(0, \infty)$ satisfying

$$
\max \left\{\mathcal{N} v_{q}(x)+(\mathscr{A}-\delta) v_{q}(x), \mathcal{M} v_{q}(x)-v_{q}(x)\right\} \leq 0, \quad x>0
$$

with the initial condition $\mathcal{M} v_{q}(0) \leq v_{q}(0)$. Then we have $v_{q}(x) \geq V_{q}(x)$.

By Lemma 2.1, we know the optimal timing of capital injection is when the surplus process hits the barrier 0 , and hence we have $\mathcal{M} V_{q}(0)=V_{q}(0)$. Furthermore, if there exists some $\eta^{*}>0$ such that $\eta^{*}=\inf \left\{x: V_{q}^{\prime}(x)=\phi\right\}$, and the value function is concave, the optimal amount of capital injection is $\eta^{*}$ and $V_{q}(0)=V_{q}\left(\eta^{*}\right)-\phi \eta^{*}-K$. Then, similar to Theorem 4.1 in Zhao et al. (2015), we give the following result. 
Lemma 4.2. Assuming that $v_{q}(x)$ is an increasing, concave and sufficiently smooth function on $(0, \infty)$, and that exists a pair $\left(\eta^{*}, x_{q}^{*}\right)$ with $0<\eta^{*}<x_{q}^{*}$, such that

$$
\begin{aligned}
& v_{q}^{\prime}\left(\eta^{*}\right)=\phi, \quad v_{q}^{\prime}\left(x_{q}^{*}\right)=1, \\
& v_{q}(0)=v_{q}\left(\eta^{*}\right)-\phi \eta^{*}-K, \\
& (\mathscr{A}-\delta) v_{q}(x)=0, \quad 0<x<x_{q}^{*}, \\
& (\mathscr{A}-\delta) v_{q}(x)+\gamma\left[x-x_{q}^{*}+v_{q}\left(x_{q}^{*}\right)-v_{q}(x)\right]=0, \quad x \geq x_{q}^{*},
\end{aligned}
$$

we have $v_{q}(x) \geq V_{q}(x)$.

Proof. By the proof of $(i)$ in Lemma 3.2, we have

$$
\mathcal{N} v_{q}(x)+(\mathscr{A}-\delta) v_{q}(x)=0, \quad x>0 .
$$

Setting $F(y)=v_{q}(x+y)-\phi y-K$, we have that the $F(y)$ is increasing on $\left[0, \eta^{*}-x\right)$ and decreasing on $\left[\eta^{*}-x, \infty\right)$. Then

$$
\mathcal{M} v_{q}(x)=\left\{\begin{array}{l}
v_{q}\left(\eta^{*}\right)-\phi\left(\eta^{*}-x\right)-K, \quad 0 \leq x<\eta^{*} \\
v_{q}(x)-K, \quad \eta^{*} \leq x<\infty
\end{array}\right.
$$

which together with $v_{q}(0)=v_{q}\left(\eta^{*}\right)-\phi \eta-K$ and $v_{q}^{\prime}(x) \geq \phi$ for $0<x \leq \eta^{*}$, we obtain $\mathcal{M} v_{q}(x) \leq v_{q}(x)$ for $x>0$ and $\mathcal{M} h(0)=h(0)$. Then $v_{q}(x)$ satisfies the conditions of Lemma 4.1, and the result is obtained.

\subsection{Optimal strategy for the Optimal Problem without Ruin}

By the discussions in Subsection 4.1 and Lemma 2.1, we construct the following strategy $\pi_{q}$, for $0<\eta<x_{q}$,

$$
\begin{aligned}
& L_{t}^{\pi_{q}}=\int_{0}^{t}\left(X_{s-}^{\pi_{q}}+\Delta X_{s}-x_{q}\right) \mathbf{1}_{\left\{X_{s-}^{\pi_{q}}+\Delta X_{s} \geq x_{q}\right\}} d N_{s}, G_{t}^{\pi_{q}}=\sum_{\tau_{n}^{\pi_{q}} \leq t} \xi_{n}^{\pi_{q}}, \\
& \xi_{n}^{\pi_{q}}=\eta, n=1,2, \cdots, \tau_{1}^{\pi_{q}}=\inf \left\{t \geq 0: X_{t-}^{\pi_{q}}=0 \text { and } \Delta X_{t}=0\right\}, \\
& \tau_{n}^{\pi_{q}}=\inf \left\{t>\tau_{n-1}^{\pi_{q}}: X_{t-}^{\pi_{q}}=0 \text { and } \Delta X_{t}=0\right\}, n=2,3, \cdots .
\end{aligned}
$$

Then the above strategy $\pi_{q} \in \Pi_{q}$, and the associated performance function $V\left(x ; \pi_{q}\right)$ is given by

$$
V\left(x ; \pi_{q}\right)=E^{x}\left[\int_{0}^{\infty} e^{-\delta s}\left(X_{s-}^{\pi_{q}}+\Delta X_{s}-x_{q}\right) \mathbf{1}_{\left\{X_{s-}^{\pi_{q}}+\Delta X_{s} \geq x_{q}\right\}} d N_{s}-\sum_{n=1}^{\infty} e^{-\delta \tau_{n}^{\pi_{q}}}(K+\phi \eta)\right] .
$$

By the same arguments of Theorem 3.1, we give the following lemma. 
Lemma 4.3. For $x \geq x_{q}$, the performance function $V\left(x ; \pi_{q}\right)$ in $(4.5)$ is given by

$$
V\left(x ; \pi_{q}\right)=\frac{\gamma}{\delta+\gamma} A_{1}\left(x-x_{q}\right)+V\left(x_{q} ; \pi_{q}\right) B_{1}\left(x-x_{q}\right)
$$

where $A_{1}(x)$ and $B_{1}(x)$ are defined by (3.18) and (3.19), respectively.

Now, we discuss $V\left(x ; \pi_{q}\right)$ in the case of $0 \leq x<x_{q}$. Let $\tilde{Y}_{t}=x_{q}-X_{t}$, and

$$
\begin{aligned}
& S_{0}^{-}=\inf \left\{t \geq 0: X_{t}<0\right\}=\inf \left\{t \geq 0: \tilde{Y}_{t}>x_{q}\right\} \triangleq \tilde{\kappa}_{x_{q}}^{+}, \\
& T_{x_{q}}^{+}=\min \left\{T_{i}: X_{T_{i}}>x_{q}, i=1,2 \cdots\right\}=\min \left\{T_{i}: \tilde{Y}_{T_{i}}<0, i=1,2 \cdots\right\} \triangleq \tilde{T}_{0}^{-},
\end{aligned}
$$

Lemma 4.4. For $0 \leq x<x_{q}$, the performance function $V\left(x ; \pi_{q}\right)$ in (4.5) is given by

$$
V\left(x ; \pi_{q}\right)=\frac{\gamma}{\delta+\gamma} A_{2}\left(x_{q}-x\right)+V\left(x_{q} ; \pi_{q}\right) B_{2}\left(x_{q}-x\right),
$$

where $A_{2}(x)$ and $B_{2}(x)$ are defined by (3.30) and (3.31), respectively.

Proof. By the strong Markov property and the law of total probability, we have

$$
\begin{aligned}
V_{q}\left(x ; \pi_{q}\right)= & E^{x}\left[e^{-\delta T_{x_{q}}^{+}}\left(X_{T_{x_{q}}^{+}}-x_{q}\right) \mathbf{1}_{\left\{T_{x_{q}}^{+}<S_{0}^{-}\right\}}\right]+V_{q}\left(x_{q} ; \pi_{q}\right) E^{x}\left[e^{-\delta T_{x_{q}}^{+}} \mathbf{1}_{\left\{T_{x_{q}}^{+}<S_{0}^{-}\right\}}\right] \\
& +E^{x}\left[e^{-\delta S_{0}^{-}} \mathbf{1}_{\left\{T_{x_{q}}^{+} \geq S_{0}^{-}\right\}}\right]\left[V_{q}\left(\eta ; \pi_{q}\right)-(\phi \eta+K)\right] .
\end{aligned}
$$

Letting $x$ go to zero in the above equation, we get

$$
V_{q}\left(0 ; \pi_{q}\right)=V_{q}\left(\eta ; \pi_{q}\right)-(\phi \eta+K) \text {. }
$$

By (12) (for a killing rate $\delta>0$ ) in Albrecher et al. (2016), we obtain

$$
E^{x}\left[e^{-\delta S_{0}^{-}} \mathbf{1}_{\left\{T_{x_{q}}^{+} \geq S_{0}^{-}\right\}}\right]=E^{x_{q}-x}\left[e^{-\delta \tilde{\kappa}_{x_{q}}^{+}} \mathbf{1}_{\left\{\tilde{T}_{0}^{-} \geq \tilde{\kappa}_{x_{q}}^{+}\right\}}\right]=\frac{Z^{(\delta)}\left(x_{q}-x, r_{0}\right)}{Z^{(\delta)}\left(x_{q}, r_{0}\right)},
$$

where $r_{0}$ is defined by (3.13). Letting $I_{3}\left(x, x_{q}\right)=\frac{Z^{(\delta)}\left(x, r_{0}\right)}{Z^{(\delta)}\left(x_{q}, r_{0}\right)}$ and combining Lemma 3.5 , we have

$$
V_{q}\left(x ; \pi_{q}\right)=I_{1}\left(x_{q}-x, x_{q}\right)+V_{q}\left(x_{q} ; \pi_{q}\right) I_{2}\left(x_{q}-x, x_{q}\right)+V_{q}\left(0 ; \pi_{q}\right) I_{3}\left(x_{q}-x, x_{q}\right) .
$$

Letting $x$ tend to $x_{q}$ in the above equation yields

$$
I_{1}\left(0, x_{q}\right)=V_{q}\left(x_{q} ; \pi_{q}\right)\left(1-I_{2}\left(0, x_{q}\right)\right)-V_{q}\left(0 ; \pi_{q}\right) I_{3}\left(0, x_{q}\right) .
$$

Replacing $x_{p}$ by $x_{q}$ in (3.33) and (3.35), we obtain the expressions of $I_{1}\left(x, x_{q}\right)$ and 
$I_{2}\left(x, x_{q}\right)$, which together with $I_{3}\left(x, x_{q}\right)=Z^{(\delta)}\left(x, r_{0}\right) I_{3}\left(0, x_{q}\right)$ and (4.8) yield

$$
\begin{aligned}
& I_{1}\left(x, x_{q}\right)+V_{q}\left(x_{q} ; \pi_{q}\right) I_{2}\left(x, x_{q}\right)+V_{q}\left(0 ; \pi_{q}\right) I_{3}\left(x, x_{q}\right) \\
= & \frac{\gamma}{\delta+\gamma} D(x)-\frac{\gamma \mu}{(\delta+\gamma)^{2}} Z^{(\delta)}\left(x, r_{0}\right)+V_{q}\left(x_{q} ; \pi_{q}\right) \frac{\gamma}{\delta+\gamma}\left(Z^{(\delta)}(x)-Z^{(\delta)}\left(x, r_{0}\right)\right) \\
& +Z^{(\delta)}\left(x, r_{0}\right)\left[I_{1}\left(0, x_{q}\right)+V_{q}\left(x_{q} ; \pi_{q}\right) I_{2}\left(0, x_{q}\right)+V_{q}\left(0 ; \pi_{q}\right) I_{3}\left(0, x_{q}\right)\right] \\
= & \frac{\gamma}{\delta+\gamma} A_{2}(x)+V_{q}\left(x_{q} ; \pi_{q}\right) B_{2}(x) .
\end{aligned}
$$

The proof is completed.

Remark 4.1. By Remark 3.2 (ii), we can show that $V\left(x ; \pi_{q}\right)$ given by (4.6) and (4.7) is increasing and sufficiently smooth on $(0, \infty)$. Furthermore, $V\left(x ; \pi_{q}\right)$ satisfies the integro-differential equations (3.8) and (3.9).

Theorem 4.1. The value function $V_{q}(x)$ in (4.1) is given by

$$
V\left(x ; \pi_{q}^{*}\right)=\left\{\begin{array}{l}
-\frac{\gamma \bar{Z}^{(\delta)}\left(x_{q}^{*}-x\right)}{\gamma+\delta}-\frac{R\left(x_{q}^{*}-x\right)}{(\gamma+\delta) r_{0}}+\frac{\gamma}{\gamma+\delta} \frac{\mu}{\delta}, 0 \leq x<x_{q}^{*} \\
-\frac{\delta}{(\gamma+\delta) r_{0}} e^{-r_{0}\left(x-x_{q}^{*}\right)}+\frac{\gamma}{\gamma+\delta}\left(x-x_{q}^{*}-\frac{1}{r_{0}}+\frac{\mu}{\delta}\right), x \geq x_{q}^{*},
\end{array}\right.
$$

where $R(x)$ is defined by (3.39), and the optimal strategy $\pi_{q}^{*}$ is given by (4.4) with the parameters $x_{q}^{*}$ and $\eta^{*}$ determined by $V^{\prime}\left(\eta^{*} ; \pi_{q}^{*}\right)=\phi$ and $V\left(\eta^{*} ; \pi_{q}^{*}\right)-V\left(0 ; \pi_{q}^{*}\right)=$ $\phi \eta^{*}+K$, i.e.,

$$
\left\{\begin{array}{l}
\frac{\gamma}{\delta+\gamma} Z^{(\delta)}\left(x_{q}^{*}-\eta^{*}\right)+\frac{\delta}{\delta+\gamma} Z^{(\delta)}\left(x_{q}^{*}-\eta^{*}, r_{0}\right)=\phi \\
\frac{\gamma}{\delta+\gamma}\left(\bar{Z}^{(\delta)}\left(x_{q}^{*}\right)-\bar{Z}^{(\delta)}\left(x_{q}^{*}-\eta^{*}\right)\right)+\frac{R\left(x_{q}^{*}\right)-R\left(x_{q}^{*}-\eta^{*}\right)}{(\delta+\gamma) r_{0}}=\phi \eta^{*}+K .
\end{array}\right.
$$

Proof. By Lemma 4.2, if the strategy $\pi_{q}^{*}$ is optimal, we have $V^{\prime}\left(x_{q}^{*} ; \pi_{q}^{*}\right)=1$. Similar to (3.37), we obtain

$$
V\left(x_{q}^{*} ; \pi_{q}^{*}\right)=\frac{\gamma}{\delta+\gamma} \frac{\mu}{\delta}-\frac{1}{r_{0}} .
$$

Substituting the above equation into (4.6) and (4.7) yields (4.9). If there exists a pair of $\left(\eta^{*}, x_{q}^{*}\right)$ satisfying the equations (4.10) and (4.11), similar to the discussions of $V\left(x ; \pi_{p}^{*}\right)$ in $(3.38)$, we can prove that $V\left(x ; \pi_{q}^{*}\right)$ in $(4.9)$ satisfies all the conditions of Lemma 4.2, and hence the results are obtained. Therefore, we only need to show that there exists a pair of $\left(\eta^{*}, x_{q}^{*}\right)$ solving (4.10) and (4.11) with $0<\eta^{*}<x_{q}^{*}$. 
For convenience, we define a function

$$
f(x)=\frac{\gamma}{\delta+\gamma} Z^{(\delta)}(x)+\frac{\delta}{\delta+\gamma} Z^{(\delta)}\left(x, r_{0}\right), \quad x \geq 0 .
$$

Making the change of variable $x=x_{q}^{*}-\eta^{*}$, we can rewrite (4.10) as $f(x)=\phi$. By (3.12) and (3.13) in Pérez and Yamazaki (2016), we have

$$
f(0)=1<\phi, \quad f^{\prime}(x)>0, x>0, \quad \lim _{x \rightarrow+\infty} f(x)=+\infty .
$$

Hence, there exists a unique $x_{1}>0$ such that $f\left(x_{1}\right)=\phi$. We define another function with respect to $x_{q}$ as follows, for $x_{q} \geq x_{1}$,

$$
f_{1}\left(x_{q}\right)=\frac{\gamma}{\delta+\gamma}\left(\bar{Z}^{(\delta)}\left(x_{q}\right)-\bar{Z}^{(\delta)}\left(x_{1}\right)\right)+\frac{R\left(x_{q}\right)-R\left(x_{1}\right)}{(\delta+\gamma) r_{0}}-\phi\left(x_{q}-x_{1}\right)-K .
$$

Then the equation (4.11) can be written as $f_{1}\left(x_{q}\right)=0$. By $R^{\prime}\left(x_{q}\right)=r_{0} \delta Z^{(\delta)}\left(x_{q}, r_{0}\right)$, we have

$$
f_{1}^{\prime}\left(x_{q}\right)=\frac{\gamma}{\delta+\gamma} Z^{(\delta)}\left(x_{q}\right)+\frac{\delta}{\delta+\gamma} Z^{(\delta)}\left(x_{q}, r_{0}\right)=f\left(x_{q}\right)-\phi>f\left(x_{1}\right)-\phi=0 .
$$

Furthermore, we obtain $\lim _{x_{q} \rightarrow+\infty} f_{1}^{\prime}\left(x_{q}\right)=+\infty$ and $f_{1}^{\prime \prime}\left(x_{q}\right)=f^{\prime}\left(x_{q}\right)>0$. Then we get $\lim _{x_{q} \rightarrow+\infty} f_{1}\left(x_{q}\right)=+\infty$. Noting $f_{1}\left(x_{1}\right)=-K<0$, we know there exists an $x_{q}^{*}>x_{1}$ such that $f_{1}\left(x_{q}^{*}\right)=0$, and so $\eta^{*}=x_{q}^{*}-x_{1}$ is also determined.

Remark 4.2. (i) By the above proof, if $K$ goes to zero, we have that $\eta^{*}$ tends to zero and that $x_{q}^{*}$ is determined by $\frac{\gamma}{\delta+\gamma} Z^{(\delta)}\left(x_{q}^{*}\right)+\frac{\delta}{\delta+\gamma} Z^{(\delta)}\left(x_{q}^{*}, r_{0}\right)=\phi$. Hence, we obtain Theorem 5.1 in Pérez and Yamazaki (2016).

(ii) Similar to Remark 3.4, we have

$$
\lim _{\gamma \rightarrow \infty} V\left(x ; \pi_{q}^{*}\right)=\left\{\begin{array}{l}
-\bar{Z}^{(\delta)}\left(\tilde{x}_{q}^{*}-x\right)+\frac{\mu}{\delta}, \quad 0 \leq x<\tilde{x}_{q}^{*}, \\
x-\tilde{x}_{q}^{*}+\frac{\mu}{\delta}, \quad x \geq \tilde{x}_{q}^{*},
\end{array}\right.
$$

where $\tilde{\eta}^{*}$ and $\tilde{x}_{q}^{*}$ are determined by

$$
\left\{\begin{array}{l}
Z^{(\delta)}\left(\tilde{x}_{q}^{*}-\tilde{\eta}^{*}\right)=\phi, \\
\bar{Z}^{(\delta)}\left(\tilde{x}_{q}^{*}\right)-\bar{Z}^{(\delta)}\left(\tilde{x}_{q}^{*}-\tilde{\eta}^{*}\right)=\phi \tilde{\eta}^{*}+K .
\end{array}\right.
$$

Furthermore, letting $K$ tend to zero, Theorem 3.1 in Bayraktar et al. (2013) is derived. 


\section{Optimal Periodic Dividend and Capital Injection Strategy}

From the definitions of $V_{p}, V_{q}$ and $V$, we can easily get the relationship

$$
V(x) \geq \max \left\{V_{p}(x), V_{q}(x)\right\}, \quad x \geq 0 .
$$

Lemma 5.1. If the function $v(x)$ satisfies one of the following two hypotheses, we have $v(x) \geq V(x)$,

(i) If $v(x)$ satisfies the conditions of Lemma 3.1 and $\mathcal{M} v(0) \leq v(0)$;

(ii) If $v(x)$ satisfies the conditions of Lemma 4.1 and $v(0) \geq 0$.

Proof. (i) If $v^{\prime}(x)<\phi$ for all $x>0$, we have $\mathcal{M} v(x)=v(x)-K<v(x)$. If there exists $\eta>0$ such that $v^{\prime}(\eta) \geq \phi$, we let $\bar{\eta}=\sup \left\{y>0: v^{\prime}(y) \geq \phi\right\}$, and then $v^{\prime}(\bar{\eta})=\phi$. Similar to (4.3), we get $\mathcal{M} v(x) \leq v(x)$. By Theorem 2.1, the result is obtained.

(ii) The result follows Theorem 2.1.

Similar to Lemma 5.2 in Zhao et al. (2015), we give the following lemma.

Lemma 5.2. For $V\left(x ; \pi_{p}^{*}\right)$ in (3.38) and $V\left(x ; \pi_{q}^{*}\right)$ in (4.9), we have

(i) $\mathcal{M V}\left(0 ; \pi_{p}^{*}\right) \leq V\left(0 ; \pi_{p}^{*}\right)$ if and only if $x_{p}^{*} \leq x_{q}^{*}$;

(ii) $V\left(0 ; \pi_{q}^{*}\right) \geq 0$ if and only if $x_{p}^{*} \geq x_{q}^{*}$.

Theorem 5.1. For the general optimal control problem in Section 2, we have

(i) If $\frac{\gamma}{\gamma+\delta} \frac{\mu}{\delta}-\frac{1}{r_{0}} \leq 0$, the value function $V(x)=V_{p}(x)=V\left(x ; \pi_{p}^{*}\right)$ is given by (3.41), and the optimal strategy $\pi^{*}$ is given by $\pi_{p}^{*}$ in (3.6) with $x_{p}^{*}=0$.

(ii) If $\frac{\gamma}{\gamma+\delta} \frac{\mu}{\delta}-\frac{1}{r_{0}}>0$ and $x_{p}^{*} \leq x_{q}^{*}$, the value function $V(x)=V_{p}(x)=V\left(x ; \pi_{p}^{*}\right)$ is given by (3.38), and the strategy $\pi^{*}$ is given by $\pi_{p}^{*}$ in (3.6) with $x_{p}^{*}>0$ determined by (3.40)

(iii) If $\frac{\gamma}{\gamma+\delta} \frac{\mu}{\delta}-\frac{1}{r_{0}}>0$ and $x_{p}^{*}>x_{q}^{*}$, the value function $V(x)=V_{q}(x)=V\left(x ; \pi_{p}^{*}\right)$ is given by (4.9), and the optimal strategy $\pi^{*}$ is given $\pi_{q}^{*}$ in (4.4) with $0<\eta^{*}<x_{q}^{*}$ determined by (4.10) and (4.11).

In other words, the value function $V(x)=\max \left\{V_{p}(x), V_{q}(x)\right\}$ and the optimal dividend barrier $x^{*}=\min \left\{x_{p}^{*}, x_{q}^{*}\right\}$.

Proof. (i) If $\frac{\gamma}{\gamma+\delta} \frac{\mu}{\delta}-\frac{1}{r_{0}} \leq 0$, we know $V\left(x ; \pi_{p}^{*}\right)$ in (3.41) satisfies $0<V^{\prime}\left(x ; \pi_{p}^{*}\right) \leq 1<$ $\phi$. Then $\mathcal{M} V\left(x ; \pi_{p}^{*}\right) \leq V\left(x ; \pi_{p}^{*}\right)$ for all $x \geq 0$, furthermore, $V\left(x ; \pi_{p}^{*}\right)$ satisfies the conditions of Theorem 2.1. The results are obtained.

The results in (ii) and (iii) are obtained by Lemma 5.1 and Lemma 5.2. 


\section{Numerical Examples}

In Egami and Yamazaki (2014), the authors considered the spectrally negative phase-type Lévy process, whose scale function admits an analytical expression; they proposed an approach to approximate the scale function for a general spectrally negative Lévy process. The numerical results of this paper are based on their approximation method. For simplicity, we discuss the cases of the Lévy process $X$ with hyperexponential and Gamma distributed compound Poisson positive jumps, respectively.

\subsection{Hyper-exponential Compound Poisson Positive Jumps}

Assume that $X$ has hyperexponential compound Poisson positive jumps, i.e.,

$$
\nu(d y)=\lambda \sum_{i=1}^{n} w_{i} \alpha_{i} e^{-\alpha_{i} y} d y, y \geq 0,
$$

where $\lambda>0,0<\alpha_{1}<\alpha_{2}<\cdots<\alpha_{n}$, and $w_{i}>0$ for $1 \leq i \leq n$ such that $\sum_{i=1}^{n} w_{i}=1$. The Laplace exponent $\psi(s)$ is then

$$
\psi(s)=\frac{\sigma^{2}}{2} s^{2}+c_{0} s+\lambda \sum_{i=1}^{n} w_{i} \frac{\alpha_{i}}{\alpha_{i}+s}-\lambda .
$$

If $\sigma>0$, the equation $\psi(s)=\delta$ has $n+2$ roots, denoted by $s_{0}=\Phi(\delta)>0$, $s_{1}, s_{2}, \cdots, s_{n+1}$, and

$$
s_{n+1}<-\alpha_{n}<s_{n}<-\alpha_{n-1}<\cdots<s_{1}<0<s_{0}<r_{0} .
$$

Here, we use the Laplace transform to analyze the results in Theorem 3.3. Let $\widehat{p}(s)$ denote the Laplace transform of the function $p(x)$. By the definitions of scale functions in Appendix B, we have, for $s>r_{0}$,

$$
\widehat{Z}^{(\delta)}(s)=\frac{\psi(s)}{s(\psi(s)-\delta)}, \widehat{Z}^{(\delta)}\left(s, r_{0}\right)=\frac{\psi(s)-\delta-\gamma}{\left(s-r_{0}\right)(\psi(s)-\delta)}, \widehat{\bar{Z}}^{(\delta)}(s)=\frac{\psi(s)}{s^{2}(\psi(s)-\delta)} .
$$

Set

$$
w(x)=-\frac{\gamma \bar{Z}^{(\delta)}(x)}{\gamma+\delta}-\frac{\delta Z^{(\delta)}\left(x, r_{0}\right)+\gamma Z^{(\delta)}(x)}{(\gamma+\delta) r_{0}}+\frac{\gamma}{\gamma+\delta} \frac{\mu}{\delta}, \quad x \geq 0 .
$$

Taking the Laplace transforms on both sides of the above equation, we obtain

$$
\widehat{w}(s)=\frac{\gamma}{\gamma+\delta} \frac{r_{0} \psi(s)}{s^{2}\left(s-r_{0}\right)(\psi(s)-\delta)}-\frac{1}{r_{0}\left(s-r_{0}\right)}+\frac{\gamma}{\gamma+\delta} \frac{\mu}{\delta} \frac{1}{s}, \quad s>r_{0} .
$$


By partial fraction decomposition and after some simplifications, we have

$$
\widehat{w}(s)=\frac{\gamma}{\gamma+\delta} \sum_{k=0}^{n+1} \frac{r_{0}}{s_{k}\left(s_{k}-r_{0}\right)} \prod_{i=1}^{n} \frac{\alpha_{i}+s_{k}}{\alpha_{i}} \prod_{j=0, j \neq k}^{n+1} \frac{s_{j}}{s_{j}-s_{k}} \frac{1}{s-s_{k}}, \quad s>r_{0} .
$$

Inverting the above Laplace transform and combining Theorem 3.3, we obtain the results in Avanzi et al. (2014).

\subsection{Gamma Distributed Compound Poisson Positive Jumps}

In the following, we assume $\nu(d y)=3 y e^{-y} d y(y \geq 0)$ to illustrate our results. That is, the size of jumps follows the $\operatorname{Gamma}(2,1)$ distribution, and the arrival times of the jumps are determined by a Poisson process with rate 3.

\section{- Influences of $\phi$ and $K$}

Let $\mu=1, \sigma=0, \delta=0.15$ and $\gamma=1$. By Theorem 3.3, we obtain $x_{p}^{*}=3.4116$ and $V_{p}(3)=3.6945$. By Table 1 ( $\uparrow$ and $\downarrow$ mean increase and decrease, respectively), the level of $x_{q}^{*}$ increases as $\phi$ or $K$ increases, while the amount of capital injection $\eta^{*}$ increases when $K$ increases or $\phi$ decreases. With the increase of $K$ or $\phi$, the optimal dividend barrier $x^{*}=\min \left\{x_{p}^{*}, x_{q}^{*}\right\}$ (boldface) switches from $x_{q}^{*}$ to $x_{p}^{*}$, i.e., the optimal strategy $\pi^{*}$ switches from $\pi_{q}^{*}$ to $\pi_{p}^{*}$. Accordingly, as $\phi$ or $K$ increases, the value function $V(3)=\max \left\{V_{p}(3), V_{q}(3)\right\}$ decreases and changes from $V_{q}(3)$ to $V_{p}(3)$. Furthermore, if $\phi=1.1$ and $K \geq 0.4162$, or $\phi \geq 1.2132$ and $K=0.2$, the optimal strategy $\pi^{*} \equiv \pi_{p}^{*}$ and $V \equiv V_{p}$, i.e. the company prefers declaring ruin to rescuing itself by capital injection whenever it is on the edge of ruin.

Table 1 Influences of $\phi$ and $K$ on $\eta^{*}, x_{q}^{*}, x_{p}^{*}, v_{q}=V_{q}(3)$ and $v_{p}=V_{p}(3)$

\begin{tabular}{|c|c|c|c|c|c|c|c|c|c|}
\hline \multicolumn{5}{|c|}{$\phi=1.1$} & \multicolumn{5}{|c|}{$K=0.2$} \\
\hline$K \uparrow$ & 0.2 & 0.3 & 0.4162 & 0.5 & $\phi \uparrow$ & 1.1 & 1.2 & 1.2132 & 1.3 \\
\hline$\eta^{* \uparrow}$ & 1.6985 & 2.0399 & 2.3592 & 2.5566 & $\eta^{*} \downarrow$ & 1.6985 & 1.5212 & 1.5025 & 1.3972 \\
\hline$x_{q}^{* \uparrow}$ & 2.7509 & 3.0923 & 3.4116 & 3.6090 & $x_{q}^{* \uparrow}$ & 2.7509 & 3.3420 & 3.4116 & 3.8348 \\
\hline$x_{p}^{*} \equiv$ & 3.4116 & 3.4116 & 3.4116 & 3.4116 & $x_{p}^{*} \equiv$ & 3.4116 & 3.4116 & 3.4116 & 3.4116 \\
\hline$v_{q \downarrow} \downarrow$ & 4.3599 & 4.0204 & 3.6945 & 3.4885 & $v_{q} \downarrow$ & 4.3599 & 3.7633 & 3.6945 & 3.2841 \\
\hline$v_{p} \equiv$ & 3.6945 & 3.6945 & 3.6945 & 3.6945 & $v_{p} \equiv$ & 3.6945 & 3.6945 & 3.6945 & 3.6945 \\
\hline
\end{tabular}

${ }^{1}$ Optimal periodic dividend barrier and value function are given in bold.

${ }^{2}$ Switch of optimal strategy and value function are given in bold and italic.

\section{- Influence of $\sigma$}

We now consider the influences of the volatility $\sigma$ on the optimal periodic dividend barriers and the associated value functions. Let $\mu=0.75, \delta=0.1, \gamma=1$, $\phi=1.1$ and $K=0.35$. By Table 2 ( $\uparrow$ and $\downarrow$ mean increase and decrease, respectively), we find that, with the increase of $\sigma$, the levels of $\eta^{*}$ and $x_{q}^{*}$ increase while the levels of $x_{p}^{*}, V_{p}(4.2)$ and $V_{q}(4.2)$ decrease. Larger volatility means higher financial 
risk. For large volatility, if the capital injection is permitted, it is better to enhance the dividend barrier to guard against risk; otherwise, it is wiser to pay dividends as soon as possible. At the same time, the higher risk results in the lower value of profit (value function). As $\sigma$ increases, the optimal dividend barrier $x^{*}$ switches from $x_{q}^{*}$ to $x_{p}^{*}$, correspondingly, the optimal strategy $\pi^{*}$ switches from $\pi_{q}^{*}$ to $\pi_{p}^{*}$. That is, if the volatility is large enough, it is more advisable to declare ruin rather than rescue the company by capital injection when the ruin occurs. In this example, when $\sigma \geq 0.5776$, the optimal strategy $\pi^{*} \equiv \pi_{p}^{*}$, i.e., the company prefers ruin to capital injection when the surplus process hits 0 .

Table 2 Influences of $\sigma$ on $\eta^{*}, x_{q}^{*}, x_{p}^{*}, v_{q}=V_{q}(4.2)$ and $v_{p}=V_{p}(4.2)$

\begin{tabular}{cccccccccc}
\hline$\sigma \uparrow$ & 0.1 & 0.2 & 0.3 & 0.4 & 0.5 & $\mathbf{0 . 5 7 7 6}$ & 0.6 & 0.7 & 0.8 \\
\hline$\eta^{*} \uparrow$ & 2.6185 & 2.6207 & 2.6243 & 2.6294 & 2.6358 & 2.6418 & 2.6437 & 2.6528 & 2.6633 \\
$x_{q}^{*} \uparrow$ & $\mathbf{4 . 1 8 3 3}$ & $\mathbf{4 . 1 8 8 0}$ & $\mathbf{4 . 1 9 5 8}$ & $\mathbf{4 . 2 0 6 8}$ & $\mathbf{4 . 2 2 0 8}$ & $\mathbf{4 . 2 3 3 7}$ & 4.2378 & 4.2577 & 4.2806 \\
$x_{p}^{*} \downarrow$ & 4.2530 & 4.2512 & 4.2482 & 4.2440 & 4.2387 & $\mathbf{4 . 2 3 3 7}$ & $\mathbf{4 . 2 3 2 1}$ & $\mathbf{4 . 2 2 4 4}$ & $\mathbf{4 . 2 1 5 4}$ \\
$v_{q} \downarrow$ & $\mathbf{4 . 9 8 1 6}$ & $\mathbf{4 . 9 7 2 8}$ & $\mathbf{4 . 9 5 8 1}$ & $\mathbf{4 . 9 3 7 6}$ & $\mathbf{4 . 9 1 1 4}$ & $\mathbf{4 . 8 8 7 2}$ & 4.8797 & 4.8424 & 4.7999 \\
$v_{p} \downarrow$ & 4.9119 & 4.9095 & 4.9057 & 4.9003 & 4.8935 & $\mathbf{4 . 8 8 7 2}$ & $\mathbf{4 . 8 8 5 3}$ & $\mathbf{4 . 8 7 5 9}$ & $\mathbf{4 . 8 6 5 2}$ \\
\hline
\end{tabular}

${ }^{1}$ Optimal periodic dividend barrier and value function are given in bold.

${ }^{2}$ Switch of optimal strategy and value function are given in bold and italic.

\section{- Influences of $\mu, \delta$ and $\gamma$}

In Fig.1, we let $\delta=0.1, \gamma=1, \sigma=0, \phi=1.15$ and $K=0.2$. Then we have $x_{p}^{*} \equiv 0$ if and only if $\mu \leq \mu_{0}=0.2338$, and $x^{*} \equiv x_{p}^{*}$ if and only if $\mu \leq \mu_{1}=0.7222$. The levels of $\eta^{*}$ and $x_{q}^{*}$ are decreasing as the drift increases. In contrast, $\mu$ has a mixed impact on the barrier $x_{p}^{*}$. On one hand, the increasing drift brings the company opportunities for profit, which requires a higher dividend barrier to delay the coming of ruin. On the other hand, larger drift brings the company more benefit, which calls for a lower barrier to distribute a greater proportion of the surplus as dividend. Fig. 1 also shows that the optimal strategy $\pi^{*}$ switches from $\pi_{p}^{*}$ to $\pi_{q}^{*}$ when $\mu$ increases. That is, the company prefers capital injection to avoid ruin when its perspective is good enough.

The influence of $\delta$ is depicted by Fig. 2 with $\mu=1, \gamma=1, \sigma=0, \phi=1.1$ and $K=0.2$. We obtain $x^{*} \equiv x_{p}^{*}$ if and only if $\delta \geq \delta_{1}=0.1868$ and $x_{p}^{*} \equiv 0$ if and only if $\delta \geq \delta_{0}=0.4646$. With the increase of $\delta$, the levels of $\eta^{*}, x_{q}^{*}$ and $x_{p}^{*}$ decrease. The larger force of interest means more impatience of managers. Then it is the sooner the better to pay out dividends, which calls for the lower barriers of dividend. The optimal strategy $\pi^{*}$ switches from $\pi_{q}^{*}$ to $\pi_{p}^{*}$ with the increase of $\delta$. That is, if $\delta$ is high enough, it is advisable to declare ruin whenever it is on the edge of ruin.

Fig. 3 and Fig.4 depict respectively the effects of $\gamma$ and $\delta$ on the minimal drift level $\mu_{0}$ such that the optimal barrier is strictly positive. For fixing $\delta=0.1$, Fig.3 shows that larger $\gamma$ results in lower $\mu_{0}$. This means that more frequent dividends 
compensate for lower expected profit $\mu$. In Fig.4, fixing $\gamma=1$ and increasing $\delta$ have a increasing impact on $\mu_{0}$, which means that more impatience of manager requires a higher expected profit. These explanations are consistent with the numerical illustrations of Figure 2 in Avanzi et al. (2014).

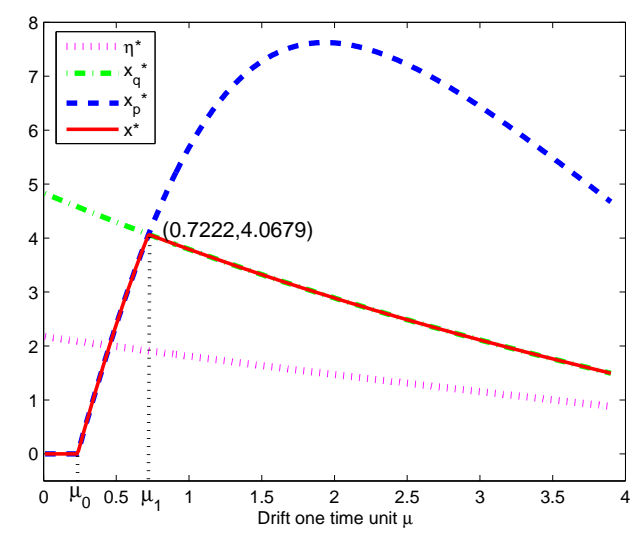

Fig. 1 Influences of $\mu$ on $\eta^{*}, x_{q}^{*}, x_{p}^{*}$ and $x^{*}$.

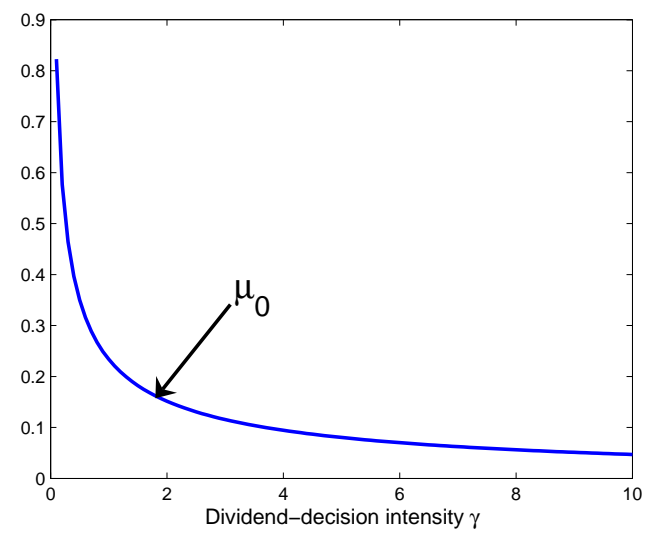

Fig. 3 Influence of $\gamma$ on minimal drift $\mu_{0}$

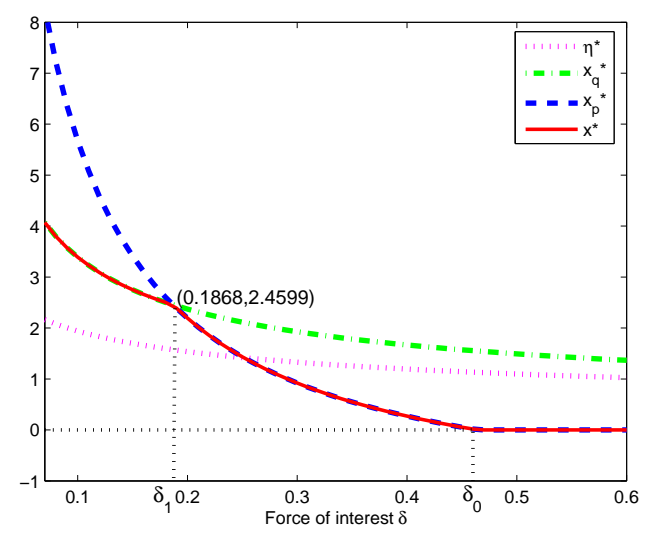

Fig. 2 Influences of $\delta$ on $\eta^{*}, x_{q}^{*}, x_{p}^{*}$ and $x^{*}$.

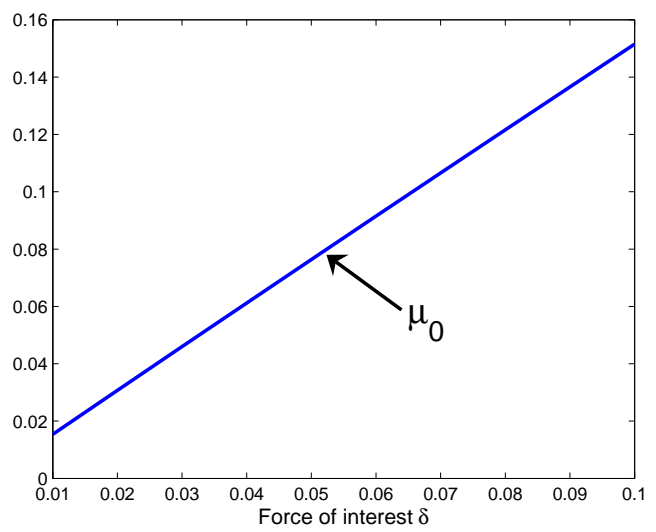

Fig. 4 Influence of $\delta$ on minimal drift $\mu_{0}$

\section{Acknowledgements}

The authors acknowledge financial support of National Natural Science Foundation of China (11501321, 11571198, 71671082, 11501319), China Postdoctoral Science Foundation (2016M592157) and Natural Science Foundation of Shandong Province in China (ZR2014AM021). 


\section{Appendix A. Proofs of Lemma 2.1 and Lemma 3.3}

Proof of Lemma 2.1. Similar to Lemma 3.2 in Peng et al. (2012), we prove the result by constructing a strategy $\hat{\pi}$ such as $V(x ; \hat{\pi}) \geq V(x ; \pi)$ where $\pi$ is an admissible strategy with the amount of capital injections $z>0$ at the initial time, i.e., $\tau_{1}^{\pi}=0$ and $\xi_{1}^{\pi}=z$.

We define the stopping time $\tau_{z}^{\pi}=\inf \left\{t \geq 0: X_{t}^{\pi} \leq z\right\}$ and the number of capital injections before $\tau_{z}^{\pi}$ by $m=\sup \left\{n \geq 1: \tau_{n}^{\pi}<\tau_{z}^{\pi}\right\}$. The strategy $\hat{\pi}$ is constructed as follows: $L_{t}^{\hat{\pi}}=L_{t}^{\pi}$ for all $t \geq 0$, and

$$
\begin{aligned}
& \tau_{i}^{\hat{\pi}}=\tau_{i+1}^{\pi}, \quad \xi_{i}^{\hat{\pi}}=\xi_{i+1}^{\pi}, \quad \text { for } i=1,2, \cdots, m-1 \\
& \tau_{i}^{\hat{\pi}}=\tau_{i}^{\pi}, \quad \xi_{i}^{\hat{\pi}}=\xi_{i}^{\pi}, \quad \text { for } \quad i=m+1, m+2, \cdots
\end{aligned}
$$

If $\tau_{z}^{\pi}=\infty$, then $\tau^{\pi}=\tau^{\hat{\pi}}=m=\infty$. We have

$$
V(x ; \hat{\pi})-V(x ; \pi)=K+\phi z \geq 0 .
$$

If $\tau_{z}^{\pi}<\infty$, we have $X_{t}^{\hat{\pi}}>0$ for $t<\tau_{z}^{\pi}$ by the definitions of $\tau_{z}^{\pi}$ and $\hat{\pi}$. We set $\tau_{m}^{\hat{\pi}}=\tau_{z}^{\pi}$ and $\xi_{m}^{\hat{\pi}}=z$, then $X_{\tau_{m}^{\hat{\pi}}}^{\hat{\pi}}=X_{\tau_{z}^{\pi}}^{\pi}$. Furthermore, we obtain

$$
V(x ; \hat{\pi})-V(x ; \pi)=K+\phi z-E^{x}\left[e^{-\delta \tau_{z}^{\pi}}(K+\phi z)\right] \geq 0 .
$$

Hence, the result is proved. In other words, if capital injection occurs, $X_{\tau_{n}-}^{\pi^{*}}=0$, a.s. holds for the optimal strategy $\pi^{*}$.

Proof of Lemma 3.3. We still denote by $S_{m}$ the stopping time $S_{m}=\inf \{t \geq$ $0: X_{t}^{\pi_{p}}>m$ or $\left.X_{t}^{\pi_{p}}<\frac{1}{m}\right\}$. Under a periodic barrier dividend strategy $\pi_{p}$ in (3.6), substituting $\nu_{s}^{\pi_{p}}=X_{s-}^{\pi_{p}}+\Delta X_{s}-x_{p}$ and $x_{p}=X_{s-}^{\pi_{p}}+\Delta X_{s}-\nu_{s}^{\pi_{p}}$ in (2.11), removing the capital injection and taking the expectations, we obtain

$$
\begin{aligned}
E^{x} & {\left[e^{-\delta\left(t \wedge S_{m}\right)} V\left(X_{t \wedge S_{m}}^{\pi_{p}} ; \pi_{p}\right)\right] } \\
= & V\left(x ; \pi_{p}\right)-E^{x}\left[\int_{0}^{t \wedge S_{m}} e^{-\delta s}\left(X_{s-}^{\pi_{p}}+\Delta X_{s}-x_{p}\right) \mathbf{1}_{\left\{X_{s-}^{\pi_{p}}+\Delta X_{s} \geq x_{p}\right\}} d N_{s}\right] \\
& +E^{x}\left\{\int_{0}^{t \wedge S_{m}}\left[(\mathscr{A}-\delta) V\left(X_{s-}^{\pi_{p}} ; \pi_{p}\right)+\gamma M(s)\right] d s\right\},
\end{aligned}
$$

where $M(s)=\left[X_{s-}^{\pi_{p}}+\Delta X_{s}-x_{p}+V\left(x_{p} ; \pi_{p}\right)-V\left(X_{s-}^{\pi_{p}}+\Delta X_{s} ; \pi_{p}\right)\right] \mathbf{1}_{\left\{X_{s-}^{\pi_{p}}+\Delta X_{s} \geq x_{p}\right\}}$. Since $V\left(x ; \pi_{p}\right)$ is bounded above by a linear function, there exist two constants $\beta_{1}$ and $\beta_{2}$ such that

$$
0 \leq e^{-\delta\left(t \wedge S_{m}\right)} V\left(X_{t \wedge S_{m}}^{\pi_{p}} ; \pi_{p}\right) \leq \beta_{1} X_{t \wedge S_{m}}^{\pi_{p}}+\beta_{2} \leq \beta_{1} \sup _{t \leq T^{\pi_{p}}} X_{t}^{\pi_{p}}+\beta_{2}
$$


By (21) in Kuznetsov et al. (2012), we have

$$
E^{x}\left[\sup _{0 \leq t \leq T_{1}} X_{t}\right]=x+E\left[\sup _{0 \leq t \leq T_{1}} X_{t}\right]=x+\frac{\mu}{\gamma}+\frac{1}{\Phi(\gamma)}
$$

where $\Phi(\gamma)=\sup \{s \geq 0: \psi(s)=\gamma\}$. Similar to (3.73) in Avanzi et al. (2014), we obtain that $\left\{e^{-\delta\left(t \wedge S_{m}\right)} V\left(X_{t \wedge S_{m}}^{\pi_{p}} ; \pi_{p}\right)\right\}$ is dominated by a nonnegative and integrable random variable. Letting $t$ and $m$ go to $\infty$ in (A.1), using the dominated convergence theorem, noting that $V\left(x ; \pi_{p}\right)$ is right continuous at zero, and $S_{m} \uparrow T^{\pi_{p}}, X_{T^{\pi_{p}}}^{\pi_{p}}=0$ a.s., we get

$$
V\left(x ; \pi_{p}\right)=E^{x}\left[\int_{0}^{T^{\pi_{p}}} e^{-\delta s}\left(X_{s-}^{\pi_{p}}+\Delta X_{s}-x_{p}\right) \mathbf{1}_{\left\{X_{s-}^{\pi_{p}}+\Delta X_{s} \geq x_{p}\right\}} d N_{s}\right],
$$

if and only if

$$
(\mathscr{A}-\delta) V\left(x ; \pi_{p}\right)+\gamma\left[x-x_{p}+V\left(x_{p} ; \pi_{p}\right)-V\left(x ; \pi_{p}\right)\right] \mathbf{1}_{\left\{x \geq x_{p}\right\}}=0 .
$$

The continuous condition in (3.10) is obtained by (3.8) and (3.9).

\section{Appendix B. Scale Functions}

We now recall the definition of the $q$-scale function for the spectrally positive Lévy process $X$, whose Laplace exponent $\psi$ is given by (2.1). For $q>0$, there exists a continuous and increasing function $W^{(q)}: \mathbb{R} \mapsto[0, \infty)$, called the $q$-scale function defined in such a way that $W^{(q)}(x)=0$ for all $x<0$ and on $[0, \infty)$ its Laplace transform is given by

$$
\int_{0}^{\infty} e^{-s x} W^{(q)}(x) d x=\frac{1}{\psi(s)-q}, \quad s>\Phi(q),
$$

where $\Phi(q)$ is given by (2.2). We give the function $Z^{(q)}(x)$, closely related to $W^{(q)}(x)$, by

$$
Z^{(q)}(x)=1+q \int_{0}^{x} W^{(q)}(y) d y, \quad x \in \mathbb{R}
$$

and its anti-derivative

$$
\bar{Z}^{(q)}(x)=\int_{0}^{x} Z^{(q)}(y) d y=x+q \int_{0}^{x} \int_{0}^{y} W^{(q)}(z) d z d y, \quad x \in \mathbb{R} .
$$

Noting that $W^{(q)}(x)$ is identically zero on the negative half line, we have $Z^{(q)}(x)=1$ and $\bar{Z}^{(q)}(x)=x$ for $x \leq 0$. 
The so-called second scale function is defined by, for $s \geq 0$,

$$
Z^{(q)}(x, s)=e^{s x}\left(1-(\psi(s)-q) \int_{0}^{x} e^{-s y} W^{(q)}(y) d y\right), \quad x \geq 0,
$$

and $Z^{(q)}(x, s)=e^{s x}$ for $x<0$. Note $Z^{(q)}(x, s)=Z^{(q)}(x)$ for $s=0$ and that we can rewrite $Z^{(q)}(x, s)$ for $s>\Phi(q)$ in the form

$$
Z^{(q)}(x, s)=(\psi(s)-q) \int_{0}^{\infty} e^{-s y} W^{(q)}(x+y) d y, \quad x \geq 0, s>\Phi(q) .
$$

From Chan et al. (2011), we know the following facts about the scale function. If $X$ has paths of bounded variation, we have that $W^{(q)} \in \mathcal{C}^{1}((0, \infty))$ if and only if the Lévy measure $\nu$ has no atoms, particularly, if $\nu$ is absolutely continuous with respect to Lebesgue measure, $W^{(q)} \in \mathcal{C}^{1}((0, \infty))$. In the case that $X$ has paths of unbounded variation, it is known that $W^{(q)} \in \mathcal{C}^{1}((0, \infty))$. Moreover, if $\sigma>0$, $\mathcal{C}^{1}((0, \infty))$ may be replaced by $\mathcal{C}^{2}((0, \infty))$. Hence, $Z^{(q)} \in \mathcal{C}^{1}((0, \infty)), \bar{Z}^{(q)} \in \mathcal{C}^{1}(\mathbb{R})$ and $\bar{Z}^{(q)} \in \mathcal{C}^{2}((0, \infty))$ for the bounded variation case, while $Z^{(q)} \in \mathcal{C}^{1}(\mathbb{R}), Z^{(q)} \in$ $\mathcal{C}^{2}((0, \infty)), \bar{Z}^{(q)} \in \mathcal{C}^{2}(\mathbb{R})$ and $\bar{Z}^{(q)} \in \mathcal{C}^{3}((0, \infty))$ for the unbounded variation case. Considering the asymptotic behavior near zero, we have

$$
W^{(q)}(0+)=\left\{\begin{array}{cl}
0, & \text { if } X \text { is of unbounded variation } \\
\frac{1}{c_{0}}, & \text { if } X \text { is of bounded variation. }
\end{array}\right.
$$

\section{References}

Albrecher, H., Cheung, E. C. K., Thonhauser, S., 2011a. Randomized observation periods for the compound poisson risk model: dividends. Astin Bulletin, 41(2), 645-672.

Albrecher, H., Gerber, H. U., Shiu, E. S., 2011b. The optimal dividend barrier in the Gamma-Omega model. European Actuarial Journal, 1(1), 43-55.

Albrecher, H., Ivanovs, J., Zhou, X., 2016. Exit identities for Lévy processes observed at Poisson arrival times. Bernoulli, 22(3), 1364-1382.

Avanzi, B., Gerber, H. U., Shiu, E. S. W., 2007. Optimal dividends in the dual model. Insurance: Mathematics and Economics, 41, 111-123.

Avanzi, B., Gerber, H. U., 2008. Optimal dividends in the dual model with diffusion. Astin Bulletin, 38(2), 653-667. 
Avanzi, B., Cheung, E. C., Wong, B., Woo, J. K., 2013. On a periodic dividend barrier strategy in the dual model with continuous monitoring of solvency. Insurance: Mathematics and Economics, 52(1), 98-113.

Avanzi, B., Shen, J., Wong, B., 2011. Optimal dividends and capital injections in the dual model with diffusion. ASTIN Bulletin, 41(2), 611-644.

Avanzi, B., Tu, V., Wong, B., 2014. On optimal periodic dividend strategies in the dual model with diffusion. Insurance: Mathematics and Economics, 55, 210-224.

Bertoin, J., 1996. Lévy processes. Cambridge Tracts in Mathematics, Cambridge University Press.

Bayraktar, E., Kyprianou, A. E., Yamazaki, K., 2013. On optimal dividends in the dual model. Astin Bulletin, 43(3), 359-372.

Bayraktar, E., Kyprianou, A., Yamazaki, K., 2014. Optimal dividends in the dual model under transaction costs. Insurance: Mathematics and Economics, 54, 133143.

Chan, T., Kyprianou, A. E., Savov, M., 2011. Smoothness of scale functions for spectrally negative Lévy processes. Probability Theory and Related Fields, 150, $129-143$.

Egami, M., Yamazaki, K., 2014. Phase-type fitting of scale functions for spectrally negative Lévy processes. Journal of Computational and Applied Mathematics, 264, 1-22.

Fleming, W. H., Soner, H. M., 2006. Controlled Markov processes and viscosity solutions (Vol. 25). Springer Science and Business Media.

Kyprianou, A., 2006. Introductory lectures on fluctuations of Lévy processes with applications. Springer Science and Business Media.

Kyprianou, A. E., Loeffen, R., Pérez, J. L., 2012. Optimal control with absolutely continuous strategies for spectrally negative Lévy processes. Journal of Applied Probability, 49(1), 150-166.

Kuznetsov, A., Kyprianou, A. E., Rivero, V., 2012. The theory of scale functions for spectrally negative Lévy processes. Lévy Matters II (pp. 97-186). Springer Berlin Heidelberg.

Peng, X., Chen, M., Guo, J., 2012. Optimal dividend and equity issuance problem with proportional and fixed transaction costs. Insurance: Mathematics and Economics, 51(3), 576-585. 
Pérez, J. L., Yamazaki, K., 2016. On the optimality of Periodic barrier strategies for a spectrally positive Lévy process. arXiv preprint arXiv: 1604.07718.

Porteus, E. L., 1977. On optimal dividend, reinvestment and liquidation policies for the firm. Operations Research, 25(5), 818-834.

Yao, D., Yang, H., Wang, R., 2011. Optimal dividend and capital injection problem in the dual model with proportional and fixed transaction costs. European Journal of Operational Research, 211(3), 568-576.

Yin, C., Wen, Y., 2013. Optimal dividends problem with a terminal value for spectrally positive Lévy processes. Insurance: Mathematics and Economics, 53(3), 769-773.

Zhao, Y., Wang, R., Yao, D., Chen, P., 2015. Optimal dividends and capital injections in the dual model with a random time horizon. Journal of Optimization Theory and Applications, 167(1), 272-295. 